\title{
Indicators for measuring health promotion practice among healthcare workers in the Nelson Mandela Bay Municipality, South Africa: A cross sectional study
}

Herbert lkechukwu Melariri ( $\square$ melariri@gmail.com )

University of KwaZulu-Natal - Howard College Campus https://orcid.org/0000-0003-0143-2692

Moses J. Chimbari

University of KwaZulu-Natal College of Health Sciences

Chester Kalinda

University of Namibia

\section{Research article}

Keywords: Health Promotion, Healthcare worker, Practice, measuring, indicators, outcome, patient

Posted Date: January 29th, 2021

DOI: https://doi.org/10.21203/rs.3.rs-154900/v1

License: () (i) This work is licensed under a Creative Commons Attribution 4.0 International License. Read Full License 


\section{Abstract}

Background: Measuring indicators for health promotion (HP) practice among healthcare workers is essential if health goals and objectives must be achieved. Such indicators provide connections between health policies and health outcomes; and ultimately add value to healthcare. This study identified indicators of health promotion among healthcare workers and compared them across levels of healthcare facilities in Nelson Mandela Bay Municipality, South Africa.

Method: A cross sectional study involving 495 healthcare workers randomly sampled from 23 hospitals including primary, secondary, and tertiary level hospitals was conducted. Questionnaires were distributed to medical doctors, nurses, and allied health workers (AHW) such as social workers, physiotherapists, occupational therapists, and speech therapist. Questions (hereto referred to as dimensions) in the questionnaires were categorized to address Facility Related Indicators (FRI), Health worker related indicators (HRI) and Outcome related indicators (ORI). Descriptive and bivariate analysis were used to identify the indicators of HP among the three HCW groups from the three levels of health care. Indicators observed to be significant in the bivariate analysis were subjected to a multivariate analysis using the multinomial regression model. $(p-v a / u e<0.05)$.

Results: Emerging Indicators were grouped into three categories: facility related indicators (FRI), healthcare workers' related indicators (HRI), and outcome related indicators (ORI). Four FRI dimensions were observed to be predictors of HP among doctors. Two dimensions were positively associated with HP practices while two others were negatively associated with HP practices among medical doctors and AHWs. On the other hand, seven HRI dimensions were significantly associated with HP among medical doctors and AHW. Furthermore, 5 outcome related indicator (ORI) dimensions were significant predictors of HP among medical doctors while only two dimensions were predictors of HP among AHW. The generalized Hosmer-Lemeshow Chi-square test showed that the models for the different HP indicators fit the data.

\section{Conclusions:}

We identified indicators for measuring HP that can be applied at primary, secondary and tertiary hospitals in Nelson Mandela Bay Municipality, South Africa. These indicators which healthcare workers and health systems' managers in the study area should be aware of may be adapted for use in other areas.

\section{Background}

Worldwide, performance measurement of health promotion (HP) practice among healthcare workers (HCWs) is a contemporary theme in health systems (1, 2). However, there is no consensus on the approach for measuring HP practice among healthcare workers. For instance, existing HP indicators focus on specific HP programmes and not on overall HP practices of healthcare workers $(3,4)$. Developing such indicators will provide the health system tools for assessing the effectiveness and quality of HP services by the HCWs. This will improve the performance of HCW to discharge HP services and ultimately reduce pressure at healthcare facilities as patients and the general community become empowered. Clearly defined indicators are critical as instruments for methodical execution of HP practices. This enhances practice objectivity, improves understanding, decision making, ensures feedback on the way things are progressing and provide for prompt warning signs to authorities (5).

Healthcare delivery faces significant challenges $(6,7)$ ranging from costs associated with healthcare to basic healthcare infrastructures. Globally, serious shortcomings have been documented across several domains in healthcare (8) and this may have long term effects on human health. Many low and middle income countries (LMIC) suffer from poor quality of care described by the World Health Organization (WHO) as worrisome particularly for Africa (9). In an analysis of healthcare services assessed against health outcomes, Africa scored an overall index of 0.48 (9) indicating that the continent utilizes only $48 \%$ of imaginable health services required for the health and well-being of its population. For instance, in Malawi, the healthcare delivery system is saddled with poor distribution of resources, disintegration of services and staff shortage (10). Similarly in Nigeria, the healthcare system is laden with poor service delivery and infrastructure (11).

In South Africa, there is a strong commitment by the state to improve the healthcare delivery system evident from supportive policies and programmes implemented; and the funding invested (12-14). Despite these efforts, health outcomes remain polarised, unequal and unfair (12). Currently the country has a two-tiered system (15) that provides services to the population - the public and the private healthcare sectors. The public health care provides free health services and is fully funded by the state hence strongly overstretched. The private healthcare sector mainly provides services to wealthier and employed classes who subscribe to private health insurance schemes $(16,17)$. This healthcare system has been described as one under increased strain as a result of overstretch of her HCWs capacity and operational resources (18).

The success of any healthcare system is largely dependent on performance of HCWs, and worldwide, millions of patients visit the healthcare facilities daily to meet the HCWs for various health needs. Establishing a set of indicators for measuring HP practices among HCWs will enhance HP awareness and practice among the HCWs. Such HP indicators will provide HCWs with vital tools for systematically conducting follow-up and have an effective HP practices (3). These tools will assist in unravelling areas for improvement and intervention. Good sets of indicators will assist policy makers and stakeholders to monitor and evaluate HP practices of HCWs. Metrics for such measurement will require clear HP indicators and a framework that integrates relationships across the different elements of HP practices. This study identified selected measures or attributes for assessing HCWs HP practices and compared them across the various levels of healthcare in the Nelson Mandela Bay Municipality, South Africa.

\section{Method}

\section{Study area}

The Nelson Mandela Bay Municipality (NMBM), is one of eight category A or metropolitan municipalities (19) in South Africa. NMBM is the largest of two Category A Metropolitan Municipalities in the Southern coast of the Eastern Cape Province (20). 
With a population of 1271 776, NMBM makes up approximately 17\% of the population of the Eastern Cape Province (20). The Municipality is comprised of the City of Port Elizabeth, Despatch (21), peri urban as well as rural areas. The population is diverse with black people being the majority, followed by coloured and whites. The NMBM has the largest economy in the province largely driven by the automotive industry and tourism sector. However, the municipality is laden with major challenges of poverty, overcrowding in townships, informal settlements, unemployment social inequalities, poor infrastructure, and poor service delivery. The Eastern Cape Province's average unemployment rate was 37.4\% in 2011 (22) and has a low life expectancy (Males - 59.6 years, females 67.1 years) compared to the national life expectancy (males 61.5 years, female - 67.7 years) (23). The major causes of death include many preventable causes. As is the case for the whole country, NMBM has primary, secondary and tertiary healthcare facilities.

\section{Study design}

A quantitative cross-sectional study was carried out between January and March 2020 in twenty-three public hospitals in NMBM. Sampled hospitals included primary, secondary, and tertiary hospitals. The study participants comprised of medical doctors, nurses, and allied health workers (AHW) (physiotherapists, occupational therapists, speech therapists, dieticians, and social workers). A sample size of 384 respondents was determined based on $95 \%$ of confidence level and an error margin of $5 \%$. The initial sample size was adjusted by $25 \%$ to 480 to cater for non-response. Questions (hereto referred to as dimensions) in the questionnaires were categorized to address Facility Related Indicators (FRI), Health worker related indicators (HRI) and Outcome related indicators (ORI). A total of 520 questionnaires were randomly distributed and 501 participants returned completed the questionnaire. Of the 501 responses, six participants did not include their professions and were therefore excluded from the final analysis.

\section{Data collection and Analysis}

A standardised and piloted questionnaire adopted from existing studies $(24,25)$, and modified to include additional questions and adapted to fit the South Africa Health system. This questionnaire was administered to HCWs from 23 health facilities in the study area. Each participant completed a self-administered semi-structured questionnaire with the following sections relating to HP and practices: sociodemographic profile of the participant(s) (sex, occupation, level of health care facility, and HP dimensions. The dimensions were categorized as Facility Related Indicators (FRI), Health worker related indicators (HRI) and Outcome related indicators (ORI). FRI dimensions included HP communication channel, coordination, HP budget, data capturing and evaluation, HP structure, quality assessment, provision to assess patients need, assessment done at first contact, documentation of HP records, availability of guidelines and policy, socio-cultural background, induction training and performance appraisal; HRI dimensions comprised - education on disease condition, guidance on diet and lifestyle, routine check-up, adequate knowledge on patients condition, fitness and health screening, assistance with welfare services, participation in training, coordinated HP training, home visits, community based placement, follow-up, patient empowerment, notification of patient on risk factors; and ORI dimensions (coverage of target population, reduction in number of deaths, reduction in number of diseases, reduction in number of injuries and disabilities, reduced inequities in health, improved need assessment and improved community mobilization and participation). The data collection process is summarized in Fig. 1.

Data collected were captured in Microsoft Excel 2016 Spreadsheet, cleaned, and analyzed using StatalC 15 (Stata Statistical Software: Release 15. College Station, TX: StataCorp LLC). Descriptive and inferential statistics were used to describe and analyze the data collected using univariate, bivariate and multivariate statistics. Descriptive statistics were used to summarize the data. Associations between categorical variables were determined using chi-square tests. In our analysis, we controlled for level of healthcare facility. HCWs were categorized as medical doctors, nurses, and allied health workers (AHW) (social workers, physiotherapists, occupational therapists, and speech therapist). To determine the influence of the indicators on the HP dimensions, a multinomial logistic model was fitted with the classification of healthcare workers (HCWs) as the dependent variable. To fit this model, all indicators that had a p-value of less than 0.1 in the Univariate model were used in the model. The fit of the final models for different HP indicators which included varied number of explanatory variables were assessed using the generalized Hosmer-Lemeshow goodness-of-fit test, chi-square test and models were regarded significant if the resulting p-value was greater than $0.05(26)$.

The Hosmer-Lemeshow goodness-of-fit and chi-square tests were employed in assessing the final model for the various HP and DP enablers.

\section{Results}

\section{Demographic characteristics}

Of the 520 health care workers (HCWs) who were enrolled into the study, only 495 participants were considered for the final analysis (Figure 1 ). Of these, $39 \%$ $(n=192)$ were medical doctors, $47 \%(n=234)$ were nurses and $14 \%(n=69)$ were allied health workers. The results further show that $26.26 \%(n=130)$ of the HCWs were from primary healthcare, $4 \%(n=17)$ were from secondary and $70.30 \%(n=348)$ were from tertiary healthcare facilities. Among these respondents, $75.42 \%(n=362)$ were females while $24.58 \%(n=118)$ were males.

\section{Facility Related Indicator (FRI)}

Fourteen dimensions were associated with health promotion practices among HCWs in the study area. These include - HP communication channel, HP coordinator, HP budget, data routinely captured on HP interventions, data available to staff for HP evaluation, HP quality assessment programme, provision to assess patient HP need, documentation of patient's HP record, guidelines for reassessing patients HP need at discharge, guidelines for reassessing patients HP need post intervention, update on patients' sociocultural background, HP policy, HP induction training for new staff, and HP performance appraisal system (See Additional file 1). When corrected for healthcare facility level, 14 dimensions were significant indicators of HP at tertiary facility level while only two dimensions were significant indicators of HP at primary healthcare level. On the other hand, none of the FRI dimension was a significant indicator of HP at secondary healthcare level (Table 1). 


\begin{tabular}{|c|c|c|c|c|c|c|c|c|c|c|c|c|c|}
\hline \multirow[t]{2}{*}{ FRI Dimensions } & \multirow[t]{2}{*}{ Profession } & \multicolumn{4}{|c|}{ Primary Healthcare Level } & \multicolumn{4}{|c|}{ Secondary Healthcare Level } & \multicolumn{4}{|c|}{ Tertiary Healthcare Level } \\
\hline & & Yes & No & $\begin{array}{l}\text { Idon't } \\
\text { Know }\end{array}$ & $\begin{array}{l}P \\
\text { value }\end{array}$ & Yes & No & $\begin{array}{l}\text { I don't } \\
\text { Know }\end{array}$ & $\begin{array}{l}\mathrm{P} \\
\text { value }\end{array}$ & Yes & No & $\begin{array}{l}\text { I don't } \\
\text { Know }\end{array}$ & $\begin{array}{l}\mathrm{P} \\
\text { value }\end{array}$ \\
\hline \multirow{3}{*}{$\begin{array}{l}\text { Any } \\
\text { communication } \\
\text { channel for HP } \\
\text { in your facility }\end{array}$} & Doctors & $\begin{array}{l}2.48 \% \\
(n=3)\end{array}$ & $\begin{array}{l}3.31 \% \\
(n=4)\end{array}$ & $\begin{array}{l}0 \% \\
(n=0\end{array}$ & \multirow[t]{3}{*}{0.447} & $\begin{array}{l}6.25 \% \\
(n=1)\end{array}$ & $\begin{array}{l}31.25 \% \\
(n=5)\end{array}$ & $\begin{array}{l}0 \% \\
(n=0\end{array}$ & \multirow[t]{3}{*}{0.117} & $\begin{array}{l}7.29 \% \\
(n=24)\end{array}$ & $\begin{array}{l}44.68 \% \\
(n=147)\end{array}$ & $0 \%(n=0$ & \multirow[t]{3}{*}{0.000} \\
\hline & Nurses & $\begin{array}{l}52.90 \% \\
(n=64)\end{array}$ & $\begin{array}{l}34.71 \% \\
(n=42)\end{array}$ & $\begin{array}{l}0 \% \\
(n=0\end{array}$ & & $\begin{array}{l}12.50 \% \\
(n=2\end{array}$ & $\begin{array}{l}6.25 \% \\
(n=1\end{array}$ & $\begin{array}{l}0 \% \\
(n=0\end{array}$ & & $\begin{array}{l}15.81 \% \\
(n=52)\end{array}$ & $\begin{array}{l}16.72 \% \\
(n=55)\end{array}$ & $0 \%(n=0$ & \\
\hline & Allied HW & $\begin{array}{l}4.96 \% \\
(n=6)\end{array}$ & $\begin{array}{l}1.65 \% \\
(n=2)\end{array}$ & $\begin{array}{l}0 \% \\
(n=0\end{array}$ & & $\begin{array}{l}31.25 \% \\
(n=5)\end{array}$ & $\begin{array}{l}12.50 \% \\
(n=2)\end{array}$ & $\begin{array}{l}0 \% \\
(n=0\end{array}$ & & $\begin{array}{l}5.17 \% \\
(n=17)\end{array}$ & $\begin{array}{l}10.33 \% \\
(n=34)\end{array}$ & $0 \%(n=0$ & \\
\hline \multirow[t]{3}{*}{ HP coordinator } & Doctors & $\begin{array}{l}0.78 \% \\
(n=1)\end{array}$ & $\begin{array}{l}1.55 \% \\
(n=2)\end{array}$ & $\begin{array}{l}3.10 \% \\
(n=4)\end{array}$ & \multirow[t]{3}{*}{0.197} & $\begin{array}{l}0 \% \\
(n=0)\end{array}$ & $\begin{array}{l}31.25 \% \\
(n=5)\end{array}$ & $\begin{array}{l}6.25 \% \\
(n=1)\end{array}$ & \multirow[t]{3}{*}{0.321} & $\begin{array}{l}3.89 \% \\
(n=13)\end{array}$ & $\begin{array}{l}19.46 \% \\
(n=65\end{array}$ & $\begin{array}{l}29.04 \% \\
(n=97)\end{array}$ & \multirow[t]{3}{*}{0.000} \\
\hline & Nurses & $\begin{array}{l}51.94 \% \\
(n=67)\end{array}$ & $\begin{array}{l}16.28 \% \\
(n=21)\end{array}$ & $\begin{array}{l}20.16 \% \\
(n=26)\end{array}$ & & $\begin{array}{l}6.25 \% \\
(n=1)\end{array}$ & $\begin{array}{l}6.25 \% \\
(n=1)\end{array}$ & $\begin{array}{l}6.25 \% \\
(n=1)\end{array}$ & & $\begin{array}{l}10.48 \% \\
(n=35)\end{array}$ & $\begin{array}{l}9.28 \% \\
(n=31)\end{array}$ & $\begin{array}{l}12.28 \% \\
(n=41)\end{array}$ & \\
\hline & Allied HW & $\begin{array}{l}3.88 \% \\
(n=5)\end{array}$ & $\begin{array}{l}0.08 \% \\
(n=1)\end{array}$ & $\begin{array}{l}1.55 \% \\
(n=2)\end{array}$ & & $\begin{array}{l}12.50 \% \\
(n=2)\end{array}$ & $\begin{array}{l}12.50 \% \\
(n=2)\end{array}$ & $\begin{array}{l}43.75 \% \\
(n=7)\end{array}$ & & $\begin{array}{l}4.49 \% \\
(n=15)\end{array}$ & $\begin{array}{l}2.40 \% \\
(n=8)\end{array}$ & $\begin{array}{l}8.68 \% \\
(n=29)\end{array}$ & \\
\hline \multirow[t]{3}{*}{ HP budget } & Doctors & $\begin{array}{l}0 \% \\
(n=0)\end{array}$ & $\begin{array}{l}12.50 \% \\
(n=2\end{array}$ & $\begin{array}{l}31.25 \% \\
(n=5)\end{array}$ & \multirow[t]{3}{*}{0.284} & $\begin{array}{l}0 \% \\
(n=0)\end{array}$ & $\begin{array}{l}18.75 \% \\
(n=3)\end{array}$ & $\begin{array}{l}18.75 \% \\
(n=3)\end{array}$ & \multirow[t]{3}{*}{0.180} & $\begin{array}{l}0.29 \% \\
(n=1)\end{array}$ & $\begin{array}{l}10.26 \% \\
(n=35)\end{array}$ & $\begin{array}{l}41.39 \% \\
(n=141)\end{array}$ & \multirow[t]{3}{*}{0.000} \\
\hline & Nurses & $\begin{array}{l}11.72 \% \\
(n=15)\end{array}$ & $\begin{array}{l}18.75 \% \\
(n=24)\end{array}$ & $\begin{array}{l}57.81 \% \\
(n=74)\end{array}$ & & $\begin{array}{l}0 \% \\
(n=0)\end{array}$ & $\begin{array}{l}0 \% \\
(n=0)\end{array}$ & $\begin{array}{l}18.75 \% \\
(n=3)\end{array}$ & & $\begin{array}{l}5.87 \% \\
(n=20)\end{array}$ & $\begin{array}{l}4.99 \% \\
(n=17)\end{array}$ & $\begin{array}{l}21.70 \% \\
(n=74)\end{array}$ & \\
\hline & Allied HW & $\begin{array}{l}0 \% \\
(n=0)\end{array}$ & $\begin{array}{l}3.13 \% \\
(n=4)\end{array}$ & $\begin{array}{l}3.13 \% \\
(n=4)\end{array}$ & & $\begin{array}{l}0 \% \\
(n=0)\end{array}$ & $\begin{array}{l}6.25 \% \\
(n=1)\end{array}$ & $\begin{array}{l}37.50 \% \\
(n=6)\end{array}$ & & $\begin{array}{l}1.47 \% \\
(n=5)\end{array}$ & $\begin{array}{l}4.11 \% \\
(n=14)\end{array}$ & $\begin{array}{l}9.97 \% \\
(n=34)\end{array}$ & \\
\hline \multirow{3}{*}{$\begin{array}{l}\text { Data routinely } \\
\text { captured on HP } \\
\text { interventions? }\end{array}$} & Doctors & $\begin{array}{l}1.56 \% \\
(n=2)\end{array}$ & $\begin{array}{l}0 \% \\
(n=0)\end{array}$ & $\begin{array}{l}3.91 \% \\
(n=5)\end{array}$ & \multirow[t]{3}{*}{0.384} & $\begin{array}{l}0 \% \\
(n=0)\end{array}$ & $\begin{array}{l}18.75 \% \\
(n=3)\end{array}$ & $\begin{array}{l}18.75 \% \\
(n=3)\end{array}$ & \multirow[t]{3}{*}{0.111} & $\begin{array}{l}1.18 \% \\
(n=4)\end{array}$ & $\begin{array}{l}15.09 \% \\
(n=51)\end{array}$ & $\begin{array}{l}33.14 \% \\
(n=122)\end{array}$ & \multirow[t]{3}{*}{0.000} \\
\hline & Nurses & $\begin{array}{l}44.53 \% \\
(n=57)\end{array}$ & $\begin{array}{l}13.28 \% \\
(n=17)\end{array}$ & $\begin{array}{l}30.47 \% \\
(n=39)\end{array}$ & & $\begin{array}{l}6.25 \% \\
(n=1)\end{array}$ & $\begin{array}{l}0 \% \\
(n=0)\end{array}$ & $\begin{array}{l}12.50 \% \\
(n=2)\end{array}$ & & $\begin{array}{l}9.76 \% \\
(n=33)\end{array}$ & $\begin{array}{l}4.14 \% \\
(n=14)\end{array}$ & $\begin{array}{l}18.05 \% \\
(n=61)\end{array}$ & \\
\hline & Allied HW & $\begin{array}{l}3.13 \% \\
(n=4)\end{array}$ & $\begin{array}{l}0.78 \% \\
(n=1)\end{array}$ & $\begin{array}{l}2.34 \% \\
(n=3)\end{array}$ & & $\begin{array}{l}0 \% \\
(n=0)\end{array}$ & $\begin{array}{l}6.25 \% \\
(n=1)\end{array}$ & $\begin{array}{l}37.50 \% \\
(n=6)\end{array}$ & & $\begin{array}{l}1.78 \% \\
(n=6)\end{array}$ & $\begin{array}{l}3.55 \% \\
(n=12)\end{array}$ & $\begin{array}{l}10.36 \% \\
(n=35)\end{array}$ & \\
\hline \multirow{3}{*}{$\begin{array}{l}\text { Data available } \\
\text { to staff for HP } \\
\text { evaluation? }\end{array}$} & Doctors & $\begin{array}{l}1.57 \% \\
(n=2)\end{array}$ & $\begin{array}{l}0.79 \% \\
(n=1)\end{array}$ & $\begin{array}{l}3.15 \% \\
(n=4)\end{array}$ & \multirow[t]{3}{*}{0.669} & $\begin{array}{l}0 \% \\
(n=0)\end{array}$ & $\begin{array}{l}18.75 \% \\
(n=3)\end{array}$ & $\begin{array}{l}18.75 \% \\
(n=3)\end{array}$ & \multirow[t]{3}{*}{0.184} & $\begin{array}{l}1.12 \% \\
(n=4)\end{array}$ & $\begin{array}{l}15.63 \% \\
(n=53)\end{array}$ & $\begin{array}{l}34.81 \% \\
(n=118)\end{array}$ & \multirow[t]{3}{*}{0.000} \\
\hline & Nurses & $\begin{array}{l}38.58 \% \\
(n=49)\end{array}$ & $\begin{array}{l}21.26 \% \\
(n=27)\end{array}$ & $\begin{array}{l}28.35 \% \\
(n=36)\end{array}$ & & $\begin{array}{l}6.25 \% \\
(n=1)\end{array}$ & $\begin{array}{l}0 \% \\
(n=0)\end{array}$ & $\begin{array}{l}12.50 \% \\
(n=2)\end{array}$ & & $\begin{array}{l}9.44 \% \\
(n=32)\end{array}$ & $\begin{array}{l}6.79 \% \\
(n=23)\end{array}$ & $\begin{array}{l}16.52 \% \\
(n=56)\end{array}$ & \\
\hline & Allied HW & $\begin{array}{l}3.15 \% \\
(n=4)\end{array}$ & $\begin{array}{l}0.79 \% \\
(n=1)\end{array}$ & $\begin{array}{l}2.36 \% \\
(n=3)\end{array}$ & & $\begin{array}{l}0 \% \\
(n=0)\end{array}$ & $\begin{array}{l}12.50 \% \\
(n=2)\end{array}$ & $\begin{array}{l}31.25 \% \\
(n=5)\end{array}$ & & $\begin{array}{l}1.77 \% \\
(n=6)\end{array}$ & $\begin{array}{l}2.95 \% \\
(n=10)\end{array}$ & $\begin{array}{l}10.91 \% \\
(n=37 \%)\end{array}$ & \\
\hline \multirow[t]{3}{*}{$\begin{array}{l}\text { HP structures } \\
\text { and facilities } \\
\text { required }\end{array}$} & Doctors & $\begin{array}{l}3.97 \% \\
(n=5)\end{array}$ & $\begin{array}{l}1.59 \% \\
(n=2)\end{array}$ & $\begin{array}{l}0 \% \\
(n=0\end{array}$ & \multirow[t]{3}{*}{0.865} & $\begin{array}{l}31.25 \% \\
(n=5)\end{array}$ & $\begin{array}{l}6.25 \% \\
(n=1)\end{array}$ & $\begin{array}{l}0 \% \\
(n=0\end{array}$ & \multirow[t]{3}{*}{0.768} & $\begin{array}{l}32.93 \% \\
(n=109)\end{array}$ & $\begin{array}{l}18.73 \% \\
(n=62)\end{array}$ & $0 \%(n=0$ & 0.356 \\
\hline & Nurses & $\begin{array}{l}58.73 \% \\
(n=74)\end{array}$ & $\begin{array}{l}29.37 \% \\
(n=37)\end{array}$ & $\begin{array}{l}0 \% \\
(n=0\end{array}$ & & $\begin{array}{l}12.50 \% \\
(n=2\end{array}$ & $\begin{array}{l}6.25 \% \\
(n=1)\end{array}$ & $\begin{array}{l}0 \% \\
(n=0\end{array}$ & & $\begin{array}{l}21.45 \% \\
(n=71)\end{array}$ & $\begin{array}{l}11.48 \% \\
(n=38)\end{array}$ & $0 \%(n=0$ & \\
\hline & Allied HW & $\begin{array}{l}4.76 \% \\
(n=6)\end{array}$ & $\begin{array}{l}1.59 \% \\
(n=2)\end{array}$ & $\begin{array}{l}0 \% \\
(n=0\end{array}$ & & $\begin{array}{l}37.50 \% \\
(n=6)\end{array}$ & $\begin{array}{l}6.25 \% \\
(n=1)\end{array}$ & $\begin{array}{l}0 \% \\
(n=0\end{array}$ & & $\begin{array}{l}11.48 \% \\
(n=38)\end{array}$ & $\begin{array}{l}3.93 \% \\
(n=13)\end{array}$ & $0 \%(n=0$ & \\
\hline $\begin{array}{l}\text { HP quality } \\
\text { assessment }\end{array}$ & Doctors & $\begin{array}{l}1.65 \% \\
(n=2)\end{array}$ & $\begin{array}{l}1.65 \% \\
(n=2)\end{array}$ & $\begin{array}{l}2.48 \% \\
(n=3)\end{array}$ & 0.052 & $\begin{array}{l}0 \% \\
(n=0)\end{array}$ & $\begin{array}{l}6.25 \% \\
(n=1)\end{array}$ & $\begin{array}{l}31.25 \% \\
(n=5)\end{array}$ & 0.432 & $\begin{array}{l}4.23 \% \\
(n=14)\end{array}$ & $\begin{array}{l}10.57 \% \\
(n=35)\end{array}$ & $\begin{array}{l}37.16 \% \\
(n=123)\end{array}$ & 0.000 \\
\hline & Nurses & $\begin{array}{l}41.32 \% \\
(n=50)\end{array}$ & $\begin{array}{l}14.88 \% \\
(n=18)\end{array}$ & $\begin{array}{l}31.40 \% \\
(n=38)\end{array}$ & & $\begin{array}{l}6.25 \% \\
(n=1)\end{array}$ & $\begin{array}{l}0 \% \\
(n=0)\end{array}$ & $\begin{array}{l}12.50 \% \\
(n=2)\end{array}$ & & $\begin{array}{l}16.31 \% \\
(n=54)\end{array}$ & $\begin{array}{l}3.93 \% \\
(n=13)\end{array}$ & $\begin{array}{l}12.39 \% \\
(n=41)\end{array}$ & \\
\hline & Allied HW & $\begin{array}{l}0.83 \% \\
(n=1)\end{array}$ & $\begin{array}{l}0 \% \\
(n=0)\end{array}$ & $\begin{array}{l}5.79 \% \\
(n=7)\end{array}$ & & $\begin{array}{l}2.48 \% \\
(n=3)\end{array}$ & $\begin{array}{l}6.25 \% \\
(n=1)\end{array}$ & $\begin{array}{l}18.75 \% \\
(n=3)\end{array}$ & & $\begin{array}{l}3.66 \% \\
(n=12)\end{array}$ & $\begin{array}{l}2.42 \% \\
(n=8)\end{array}$ & $\begin{array}{l}9.37 \% \\
(n=31)\end{array}$ & \\
\hline $\begin{array}{l}\text { Provision to } \\
\text { access patients }\end{array}$ & Doctors & $\begin{array}{l}2.48 \% \\
(n=3)\end{array}$ & $\begin{array}{l}3.31 \% \\
(n=4)\end{array}$ & $\begin{array}{l}0 \% \\
(n=0\end{array}$ & 0.137 & $\begin{array}{l}12.50 \% \\
(n=2)\end{array}$ & $\begin{array}{l}25.00 \% \\
(n=4)\end{array}$ & $\begin{array}{l}0 \% \\
(n=0\end{array}$ & 0.149 & $\begin{array}{l}5.41 \% \\
(n=49)\end{array}$ & $\begin{array}{l}37.11 \% \\
(n=118)\end{array}$ & $\begin{array}{l}0.31 \% \\
(n=1)\end{array}$ & 0.000 \\
\hline & Nurses & $\begin{array}{l}49.59 \% \\
(n=60)\end{array}$ & $\begin{array}{l}21.49 \% \\
(n=26)\end{array}$ & $\begin{array}{l}0 \% \\
(n=0\end{array}$ & & $\begin{array}{l}12.50 \% \\
(n=2)\end{array}$ & $\begin{array}{l}6.25 \% \\
(n=1)\end{array}$ & $\begin{array}{l}0 \% \\
(n=0\end{array}$ & & $\begin{array}{l}23.27 \% \\
(n=74)\end{array}$ & $\begin{array}{l}10.06 \% \\
(n=32)\end{array}$ & $\begin{array}{l}0.31 \% \\
(n=1)\end{array}$ & \\
\hline & Allied HW & $\begin{array}{l}4.13 \% \\
(n=5)\end{array}$ & $\begin{array}{l}2.48 \% \\
(n=3)\end{array}$ & $\begin{array}{l}0 \% \\
(n=0\end{array}$ & & $\begin{array}{l}37.50 \% \\
(n=6)\end{array}$ & $\begin{array}{l}6.25 \% \\
(n=1)\end{array}$ & $\begin{array}{l}0 \% \\
(n=0\end{array}$ & & $\begin{array}{l}5.66 \% \\
(n=18)\end{array}$ & $\begin{array}{l}7.86 \% \\
(n=25)\end{array}$ & $\begin{array}{l}0 \% \\
(n=0)\end{array}$ & \\
\hline $\begin{array}{l}\text { HP need } \\
\text { assessment }\end{array}$ & Doctors & $\begin{array}{l}5.00 \% \\
(n=4)\end{array}$ & $\begin{array}{l}0 \% \\
(n=0)\end{array}$ & $\begin{array}{l}0 \% \\
(n=0\end{array}$ & 0.562 & $\begin{array}{l}11.11 \% \\
(n=1)\end{array}$ & $\begin{array}{l}11.11 \% \\
(n=1)\end{array}$ & $\begin{array}{l}0 \% \\
(n=0\end{array}$ & 0.347 & $\begin{array}{l}20.00 \% \\
(n=33)\end{array}$ & $\begin{array}{l}21.21 \% \\
(n=35)\end{array}$ & $\begin{array}{l}0 \% \\
(n=0)\end{array}$ & 0.549 \\
\hline $\begin{array}{l}\text { contact with } \\
\text { hospital }\end{array}$ & Nurses & $\begin{array}{l}70.00 \% \\
(n=56)\end{array}$ & $\begin{array}{l}20.00 \% \\
(n=16)\end{array}$ & $\begin{array}{l}0 \% \\
(n=0\end{array}$ & & $\begin{array}{l}22.22 \% \\
(n=2)\end{array}$ & $\begin{array}{l}0 \% \\
(n=0)\end{array}$ & $\begin{array}{l}0 \% \\
(n=0\end{array}$ & & $\begin{array}{l}24.24 \% \\
(n=40)\end{array}$ & $\begin{array}{l}20.60 \% \\
(n=34)\end{array}$ & $\begin{array}{l}1.12 \% \\
(n=2)\end{array}$ & \\
\hline & Allied HW & $\begin{array}{l}3.75 \% \\
(n=3)\end{array}$ & $\begin{array}{l}1.25 \% \\
(n=1)\end{array}$ & $\begin{array}{l}0 \% \\
(n=0\end{array}$ & & $\begin{array}{l}22.22 \% \\
(n=2)\end{array}$ & $\begin{array}{l}33.33 \% \\
(n=3)\end{array}$ & $\begin{array}{l}0 \% \\
(n=0\end{array}$ & & $\begin{array}{l}7.27 \% \\
(n=12)\end{array}$ & $\begin{array}{l}5.45 \% \\
(n=9)\end{array}$ & $\begin{array}{l}0 \% \\
(n=0)\end{array}$ & \\
\hline
\end{tabular}




\begin{tabular}{|c|c|c|c|c|c|c|c|c|c|c|c|c|c|}
\hline \multirow{3}{*}{$\begin{array}{l}\text { Documentation } \\
\text { of patient's HP } \\
\text { record }\end{array}$} & Doctors & $\begin{array}{l}0.83 \% \\
(n=1)\end{array}$ & $\begin{array}{l}2.48 \% \\
(n=3)\end{array}$ & $\begin{array}{l}2.48 \% \\
(n=3)\end{array}$ & \multirow[t]{3}{*}{0.061} & $\begin{array}{l}0 \% \\
(n=0)\end{array}$ & $\begin{array}{l}18.75 \% \\
(n=3)\end{array}$ & $\begin{array}{l}18.75 \% \\
(n=3)\end{array}$ & \multirow[t]{3}{*}{0.193} & $\begin{array}{l}10.61 \% \\
(n=35)\end{array}$ & $\begin{array}{l}20.30 \% \\
(n=67)\end{array}$ & $\begin{array}{l}20.61 \% \\
(n=68)\end{array}$ & \multirow[t]{3}{*}{0.000} \\
\hline & Nurses. & $\begin{array}{l}54.55 \% \\
(n=66)\end{array}$ & $\begin{array}{l}10.74 \% \\
(n=13)\end{array}$ & $\begin{array}{l}22.31 \% \\
(n=27)\end{array}$ & & $\begin{array}{l}12.50 \% \\
(n=2)\end{array}$ & $\begin{array}{l}0 \% \\
(n=0)\end{array}$ & $\begin{array}{l}6.25 \% \\
(n=1)\end{array}$ & & $\begin{array}{l}18.49 \% \\
(n=61)\end{array}$ & $\begin{array}{l}6.67 \% \\
(n=22)\end{array}$ & $\begin{array}{l}7.58 \% \\
(n=25)\end{array}$ & \\
\hline & Allied HW & $\begin{array}{l}19.01 \% \\
(n=23)\end{array}$ & $\begin{array}{l}1.65 \% \\
(n=2)\end{array}$ & $\begin{array}{l}2.48 \% \\
(n=3)\end{array}$ & & $\begin{array}{l}18.75 \% \\
(n=3)\end{array}$ & $\begin{array}{l}6.25 \% \\
(n=1)\end{array}$ & $\begin{array}{l}18.75 \% \\
(n=3)\end{array}$ & & $\begin{array}{l}4.55 \% \\
(n=15)\end{array}$ & $\begin{array}{l}4.85 \% \\
(n=16)\end{array}$ & $\begin{array}{l}6.36 \% \\
(n=21)\end{array}$ & \\
\hline \multirow{3}{*}{$\begin{array}{l}\text { Guidelines for } \\
\text { reassessing } \\
\text { patients HP } \\
\text { needs at } \\
\text { discharge }\end{array}$} & Doctors & $\begin{array}{l}0.84 \% \\
(n=1)\end{array}$ & $\begin{array}{l}2.52 \% \\
(n=3)\end{array}$ & $\begin{array}{l}2.52 \% \\
(n=3)\end{array}$ & \multirow[t]{3}{*}{0.228} & $\begin{array}{l}0 \% \\
(n=0)\end{array}$ & $\begin{array}{l}18.75 \% \\
(n=3)\end{array}$ & $\begin{array}{l}18.75 \% \\
(n=3)\end{array}$ & \multirow[t]{3}{*}{0.059} & $\begin{array}{l}6.27 \% \\
(n=21)\end{array}$ & $\begin{array}{l}19.10 \% \\
(n=64)\end{array}$ & $\begin{array}{l}25.97 \% \\
(n=87)\end{array}$ & \multirow[t]{3}{*}{0.000} \\
\hline & Nurses & $\begin{array}{l}34.45 \% \\
(n=41)\end{array}$ & $\begin{array}{l}15.13 \% \\
(n=18)\end{array}$ & $\begin{array}{l}38.66 \% \\
(n=46)\end{array}$ & & $\begin{array}{l}12.50 \% \\
(n=2)\end{array}$ & $\begin{array}{l}6.25 \% \\
(n=1)\end{array}$ & $\begin{array}{l}0 \% \\
(n=0)\end{array}$ & & $\begin{array}{l}17.02 \% \\
(n=57)\end{array}$ & $\begin{array}{l}7.46 \% \\
(n=25)\end{array}$ & $\begin{array}{l}8.66 \% \\
(n=29)\end{array}$ & \\
\hline & Allied HW & $\begin{array}{l}1.68 \% \\
(n=2)\end{array}$ & $\begin{array}{l}2.52 \% \\
(n=3)\end{array}$ & $\begin{array}{l}1.68 \% \\
(n=2)\end{array}$ & & $\begin{array}{l}12.50 \% \\
(n=2)\end{array}$ & $\begin{array}{l}0 \% \\
(n=0)\end{array}$ & $\begin{array}{l}31.25 \% \\
(n=5)\end{array}$ & & $\begin{array}{l}1.79 \% \\
(n=6 \%)\end{array}$ & $\begin{array}{l}4.18 \% \\
(n=14)\end{array}$ & $\begin{array}{l}9.55 \% \\
(n=32)\end{array}$ & \\
\hline \multirow{3}{*}{$\begin{array}{l}\text { Guidelines for } \\
\text { reassessing } \\
\text { patients HP } \\
\text { needs post } \\
\text { intervention }\end{array}$} & Doctors & $\begin{array}{l}1.67 \% \\
(n=2)\end{array}$ & $\begin{array}{l}1.67 \% \\
(n=2)\end{array}$ & $\begin{array}{l}2.50 \% \\
(n=3)\end{array}$ & \multirow[t]{3}{*}{0.835} & $\begin{array}{l}0 \% \\
(n=0)\end{array}$ & $\begin{array}{l}18.75 \% \\
(n=3)\end{array}$ & $\begin{array}{l}18.75 \% \\
(n=3)\end{array}$ & \multirow[t]{3}{*}{0.328} & $\begin{array}{l}6.97 \% \\
(n=23)\end{array}$ & $\begin{array}{l}19.09 \% \\
(n=63)\end{array}$ & $\begin{array}{l}26.36 \% \\
(n=87)\end{array}$ & \multirow[t]{3}{*}{0.000} \\
\hline & Nurses & $\begin{array}{l}37.50 \% \\
(n=45)\end{array}$ & $\begin{array}{l}13.33 \% \\
(n=16)\end{array}$ & $\begin{array}{l}36.67 \% \\
(n=44)\end{array}$ & & $\begin{array}{l}6.25 \% \\
(n=1)\end{array}$ & $\begin{array}{l}0 \% \\
(n=0)\end{array}$ & $\begin{array}{l}12.50 \% \\
(n=2)\end{array}$ & & $\begin{array}{l}16.06 \% \\
(n=53)\end{array}$ & $\begin{array}{l}8.18 \% \\
(n=27)\end{array}$ & $\begin{array}{l}7.88 \% \\
(n=26)\end{array}$ & \\
\hline & Allied HW & $\begin{array}{l}2.50 \% \\
(n=3)\end{array}$ & $\begin{array}{l}1.67 \% \\
(n=2)\end{array}$ & $\begin{array}{l}2.50 \% \\
(n=3)\end{array}$ & & $\begin{array}{l}6.25 \% \\
(n=1)\end{array}$ & $\begin{array}{l}6.25 \% \\
(n=1)\end{array}$ & $\begin{array}{l}31.25 \% \\
(n=5)\end{array}$ & & $\begin{array}{l}1.81 \% \\
(n=6)\end{array}$ & $\begin{array}{l}4.85 \% \\
(n=16)\end{array}$ & $\begin{array}{l}8.79 \% \\
(n=29)\end{array}$ & \\
\hline \multirow{3}{*}{$\begin{array}{l}\text { Update on } \\
\text { patient's socio- } \\
\text { cultural } \\
\text { background }\end{array}$} & Doctors & $\begin{array}{l}0.81 \% \\
(n=1)\end{array}$ & $\begin{array}{l}2.44 \% \\
(n=3)\end{array}$ & $\begin{array}{l}2.44 \% \\
(n=3)\end{array}$ & \multirow[t]{3}{*}{0.020} & $\begin{array}{l}6.25 \% \\
(n=1)\end{array}$ & $\begin{array}{l}12.50 \% \\
(n=2)\end{array}$ & $\begin{array}{l}18.75 \% \\
(n=3)\end{array}$ & \multirow[t]{3}{*}{0.842} & $\begin{array}{l}15.38 \% \\
(n=52)\end{array}$ & $\begin{array}{l}15.98 \% \\
(n=54)\end{array}$ & $\begin{array}{l}19.82 \% \\
(n=67)\end{array}$ & \multirow[t]{3}{*}{0.000} \\
\hline & Nurses & $\begin{array}{l}57.72 \% \\
(n=71)\end{array}$ & $\begin{array}{l}15.45 \% \\
(n=19)\end{array}$ & $\begin{array}{l}14.63 \% \\
(n=18)\end{array}$ & & $\begin{array}{l}6.25 \% \\
(n=1)\end{array}$ & $\begin{array}{l}6.25 \% \\
(n=1)\end{array}$ & $\begin{array}{l}6.25 \% \\
(n=1)\end{array}$ & & $\begin{array}{l}24.56 \% \\
(n=83)\end{array}$ & $\begin{array}{l}4.73 \% \\
(n=16)\end{array}$ & $\begin{array}{l}3.85 \% \\
(n=13)\end{array}$ & \\
\hline & Allied HW & $\begin{array}{l}2.44 \% \\
(n=3)\end{array}$ & $\begin{array}{l}3.25 \% \\
(n=4)\end{array}$ & $\begin{array}{l}0.81 \% \\
(n=1)\end{array}$ & & $\begin{array}{l}18.75 \% \\
(n=3)\end{array}$ & $\begin{array}{l}6.25 \% \\
(n=1)\end{array}$ & $\begin{array}{l}18.75 \% \\
(n=3)\end{array}$ & & $\begin{array}{l}10.36 \% \\
(n=35)\end{array}$ & $\begin{array}{l}0.89 \% \\
(n=3)\end{array}$ & $\begin{array}{l}4.44 \% \\
(n=15)\end{array}$ & \\
\hline \multirow[t]{3}{*}{$\begin{array}{l}\text { Any health } \\
\text { promotion } \\
\text { policy }\end{array}$} & Doctors & $\begin{array}{l}0 \% \\
(n=0)\end{array}$ & $\begin{array}{l}1.63 \% \\
(n=2)\end{array}$ & $\begin{array}{l}4.07 \% \\
(n=5)\end{array}$ & 0.034 & $\begin{array}{l}6.25 \% \\
(n=1)\end{array}$ & $\begin{array}{l}6.25 \% \\
(n=1)\end{array}$ & $\begin{array}{l}25 \% \\
(n=4)\end{array}$ & \multirow[t]{3}{*}{0.683} & $\begin{array}{l}4.49 \% \\
(n=15)\end{array}$ & $\begin{array}{l}8.08 \% \\
(n=27)\end{array}$ & $\begin{array}{l}39.82 \% \\
(n=133)\end{array}$ & \multirow[t]{3}{*}{0.000} \\
\hline & Nurses & $\begin{array}{l}41.46 \% \\
(n=51)\end{array}$ & $\begin{array}{l}4.88 \% \\
(n=6)\end{array}$ & $\begin{array}{l}41.46 \% \\
(n=51)\end{array}$ & & $\begin{array}{l}6.25 \% \\
(n=1)\end{array}$ & $\begin{array}{l}0 \% \\
(n=0)\end{array}$ & $\begin{array}{l}12.50 \% \\
(n=2)\end{array}$ & & $\begin{array}{l}16.47 \% \\
(n=55)\end{array}$ & $\begin{array}{l}1.80 \% \\
(n=6)\end{array}$ & $\begin{array}{l}13.77 \% \\
(n=46)\end{array}$ & \\
\hline & Allied HW & $\begin{array}{l}2.44 \% \\
(n=3)\end{array}$ & $\begin{array}{l}0 \% \\
(n=0)\end{array}$ & $\begin{array}{l}4.07 \% \\
(n=5)\end{array}$ & & $\begin{array}{l}6.25 \% \\
(n=1)\end{array}$ & $\begin{array}{l}0 \% \\
(n=0)\end{array}$ & $\begin{array}{l}37.50 \% \\
(n=6)\end{array}$ & & $\begin{array}{l}2.10 \% \\
(n=7)\end{array}$ & $\begin{array}{l}2.10 \% \\
(n=7)\end{array}$ & $\begin{array}{l}11.38 \% \\
(n=38)\end{array}$ & \\
\hline \multirow{3}{*}{$\begin{array}{l}\text { HP induction } \\
\text { training for new } \\
\text { staff }\end{array}$} & Doctors & $\begin{array}{l}1.61 \% \\
(n=2)\end{array}$ & $\begin{array}{l}1.61 \% \\
(n=2)\end{array}$ & $\begin{array}{l}2.42 \% \\
(n=3)\end{array}$ & \multirow[t]{3}{*}{0.468} & $\begin{array}{l}0 \% \\
(n=0)\end{array}$ & $\begin{array}{l}18.75 \% \\
(n=3)\end{array}$ & $\begin{array}{l}18.75 \% \\
(n=3)\end{array}$ & \multirow[t]{3}{*}{0.251} & $\begin{array}{l}2.70 \% \\
(n=9)\end{array}$ & $\begin{array}{l}21.62 \% \\
(n=72)\end{array}$ & $\begin{array}{l}28.23 \% \\
(n=94)\end{array}$ & \multirow[t]{3}{*}{0.000} \\
\hline & Nurses & $\begin{array}{l}36.29 \% \\
(n=45)\end{array}$ & $\begin{array}{l}19.35 \% \\
(n=24)\end{array}$ & $\begin{array}{l}32.26 \% \\
(n=40)\end{array}$ & & $\begin{array}{l}12.50 \% \\
(n=2)\end{array}$ & $\begin{array}{l}0 \% \\
(n=0)\end{array}$ & $\begin{array}{l}6.25 \% \\
(n=1)\end{array}$ & & $\begin{array}{l}21.62 \% \\
(n=72)\end{array}$ & $\begin{array}{l}3.00 \% \\
(n=10)\end{array}$ & $\begin{array}{l}7.51 \% \\
(n=25)\end{array}$ & \\
\hline & Allied HW & $\begin{array}{l}1.61 \% \\
(n=2)\end{array}$ & $\begin{array}{l}3.23 \% \\
(n=4)\end{array}$ & $\begin{array}{l}1.61 \% \\
(n=2)\end{array}$ & & $\begin{array}{l}12.50 \% \\
(n=2)\end{array}$ & $\begin{array}{l}12.50 \% \\
(n=2)\end{array}$ & $\begin{array}{l}18.75 \% \\
(n=3)\end{array}$ & & $\begin{array}{l}3.90 \% \\
(n=13)\end{array}$ & $\begin{array}{l}4.50 \% \\
(n=15)\end{array}$ & $\begin{array}{l}6.91 \% \\
(n=23)\end{array}$ & \\
\hline \multirow{3}{*}{$\begin{array}{l}\text { HP } \\
\text { performance } \\
\text { appraisal } \\
\text { system }\end{array}$} & Doctors & $\begin{array}{l}0.81 \% \\
(n=1)\end{array}$ & $\begin{array}{l}3.25 \% \\
(n=4)\end{array}$ & $\begin{array}{l}1.63 \% \\
(n=2)\end{array}$ & \multirow[t]{3}{*}{0.151} & $\begin{array}{l}0 \% \\
(n=0)\end{array}$ & $\begin{array}{l}18.75 \% \\
(n=3)\end{array}$ & $\begin{array}{l}18.75 \% \\
(n=3)\end{array}$ & \multirow[t]{3}{*}{0.773} & $\begin{array}{l}2.40 \% \\
(n=8)\end{array}$ & $\begin{array}{l}18.62 \% \\
(n=62)\end{array}$ & $\begin{array}{l}31.53 \% \\
(n=105)\end{array}$ & \multirow[t]{3}{*}{0.000} \\
\hline & Nurses & $\begin{array}{l}34.96 \% \\
(n=43)\end{array}$ & $\begin{array}{l}21.95 \% \\
(n=27)\end{array}$ & $\begin{array}{l}30.89 \% \\
(n=38)\end{array}$ & & $\begin{array}{l}0 \% \\
(n=0)\end{array}$ & $\begin{array}{l}6.25 \% \\
(n=1)\end{array}$ & $\begin{array}{l}12.50 \% \\
(n=2)\end{array}$ & & $\begin{array}{l}17.11 \% \\
(n=57)\end{array}$ & $\begin{array}{l}5.71 \% \\
(n=19)\end{array}$ & $\begin{array}{l}9.31 \% \\
(n=31)\end{array}$ & \\
\hline & Allied HW & $\begin{array}{l}0.81 \% \\
(n=1)\end{array}$ & $\begin{array}{l}1.61 \% \\
(n=2)\end{array}$ & $\begin{array}{l}4.07 \% \\
(n=5)\end{array}$ & & $\begin{array}{l}6.25 \% \\
(n=1)\end{array}$ & $\begin{array}{l}12.50 \% \\
(n=2)\end{array}$ & $\begin{array}{l}25.00 \% \\
(n=4)\end{array}$ & & $\begin{array}{l}2.10 \% \\
(n=7)\end{array}$ & $\begin{array}{l}3.00 \% \\
(n=10)\end{array}$ & $\begin{array}{l}10.21 \% \\
(n=34)\end{array}$ & \\
\hline
\end{tabular}

Results obtained from a multinomial regression indicated that 6 dimensions were significantly associated with HP among health workers (Table 2). For medical doctors, four dimensions were observed to be significant in the final adjusted model. FRI dimensions observed to be predictors of HP among doctors were HP data available for evaluation (Coeff: $0.988,95 \% \mathrm{Cl}: 0.369-1.607)$ and quality assessment program (Coeff: $0.787,95 \% \mathrm{Cl}$ : 0.208 - 1.366,) which were observe to be 0.99 and 0.79 times respectively, higher compared to nurses. Other FRI dimensions - Provision to access patients HP need (Coeff: -2.695, $95 \%$ Cl: -3.942 - -1.448) and HP need assessment done at first contact with the hospital (Coeff: $-0.751,95 \% \mathrm{Cl}:-1.444--0.057$,) were observed to be negative predictors of HP among medical doctors (Table 2).

HP data available for evaluation (Coeff: $0.954,95 \%$ Cl: 0.149 - 1.759) and HP structures and facilities required (Coeff:1.787, 95\% Cl: 0.488 - 3.087$)$ were positively associated with HP among AHW. These factors were 0.75 and 1.8 times higher compared to nurses, respectively. On the other hand, HP Budget 
(Coeff: $-0.726,95 \% \mathrm{Cl}:-1.361$ - -0.091) and Provision to access patients HP need (Coeff: $-1.798,95 \%$ Cl: -3.428 - -0.167 ) were negatively associated with HP practices among AHW (Table 2). The generalized Hosmer-Lemeshow test Chi-square was 16.13 with p-value 0.444 , indicating a good fit of the final model.

Table 2: Facility Related Indicators' dimension associated with Health Promotion in a multivariate analysis

\begin{tabular}{|c|c|c|c|c|c|}
\hline FRI dimensions & Professions & $\begin{array}{l}\text { Coeff } \\
\text { (unadjusted) }\end{array}$ & $\begin{array}{l}95 \% \text { conf. } \\
\text { interval }\end{array}$ & $\begin{array}{l}\text { Coeff } \\
\text { (adjusted) }\end{array}$ & $\begin{array}{l}95 \% \text { conf. } \\
\text { interval }\end{array}$ \\
\hline \multirow[t]{3}{*}{ HP Budget } & $\begin{array}{l}\text { Nurses (base } \\
\text { outcome) }\end{array}$ & & & & \\
\hline & Doctors & 0.293 & $-0.364-0.950$ & 0.163 & $-0.422-0.747$ \\
\hline & Allied workers & -0.805 & $-1.524--0.086$ & -0.726 & $-1.361--0.091$ \\
\hline \multirow[t]{3}{*}{ HP data available for evaluation } & $\begin{array}{l}\text { Nurses (base } \\
\text { outcome) }\end{array}$ & & & & \\
\hline & Doctors & 0.886 & $0.235-1.537$ & 0.988 & $0.369-1.608$ \\
\hline & Allied workers & 1.113 & $0.205-2.022$ & 0.954 & $0.149-1.759$ \\
\hline \multirow[t]{3}{*}{ HP Structures \& Facilities required } & $\begin{array}{l}\text { Nurses (base } \\
\text { outcome) }\end{array}$ & & & & \\
\hline & Doctors & 0.944 & $0.122-1.765$ & 0.563 & $-0.179-1.305$ \\
\hline & Allied workers & 1.742 & $0.404-3.081$ & 1.787 & $0.488-3.087$ \\
\hline \multirow[t]{3}{*}{ Quality assessment program } & $\begin{array}{l}\text { Nurses (base } \\
\text { outcome) }\end{array}$ & & & & \\
\hline & Doctors & 0.642 & $0.001-1.283$ & 0.787 & $0.208-1.366$ \\
\hline & Allied workers & 0.513 & $-0.248-1.274$ & 0.573 & $-0.143-1.289$ \\
\hline \multirow[t]{3}{*}{ Prov to access HP need } & $\begin{array}{l}\text { Nurses (base } \\
\text { outcome) }\end{array}$ & & & & \\
\hline & Doctors & -2.888 & $-4.244--1.532$ & -2.695 & $-3.942--1.448$ \\
\hline & Allied workers & -1.814 & $-3.566-0.063$ & -1.798 & $-3.428--0.167$ \\
\hline \multirow[t]{3}{*}{$\begin{array}{l}\text { HP need assessment done at first contact with the } \\
\text { hospital }\end{array}$} & $\begin{array}{l}\text { Nurses (base } \\
\text { outcome) }\end{array}$ & & & & \\
\hline & Doctors & -0.675 & $-1.409-0.060$ & -0.751 & $-1.444--0.057$ \\
\hline & Allied workers & -0.814 & $-1.766-0.133$ & -0.789 & $-1.679-0.099$ \\
\hline
\end{tabular}

\section{Health workers Related Indicator (HRI)}

Ten dimensions comprising - education on disease condition, guidance on diet \& lifestyle, need for routine check-up, adequate knowledge on patient condition, fitness and health screening, assist with welfare services, participation in HP training, coordinated HP training for staff, home visit, community-based placements, and follow up post discharge were associated with HP practice among HCWs (See Additional file 2). At health facility level, eight dimensions were statistically significant indicators of HP at tertiary level, three at the secondary level, and only one at the primary level (Table 3). The indicator dimensions that were statistically significant at the different healthcare levels are listed in Table 3.

Table 3: Correlations of Health workers Related Indicator Dimensions with level of health care Facility 


\begin{tabular}{|c|c|c|c|c|c|c|c|c|c|c|c|c|c|}
\hline \multirow{2}{*}{$\begin{array}{l}\text { HRI } \\
\text { Dimensions }\end{array}$} & \multirow[t]{2}{*}{ Responses } & \multicolumn{4}{|c|}{ Primary Health Care Level } & \multicolumn{4}{|c|}{ Secondary Health Care level } & \multicolumn{4}{|c|}{ Tertiary Health care level } \\
\hline & & Doctors & Nurses & AHWs & $\begin{array}{l}\mathrm{P} \text { - } \\
\text { value }\end{array}$ & Doctors & Nurses & AHWs & $\begin{array}{l}\mathrm{P} \text { - } \\
\text { value }\end{array}$ & Doctors & Nurses & AHWs & $\begin{array}{l}\mathrm{P}- \\
\text { value }\end{array}$ \\
\hline \multirow{5}{*}{$\begin{array}{l}\text { Educate on } \\
\text { disease } \\
\text { condition }\end{array}$} & $\begin{array}{l}\text { Strongly } \\
\text { disagree }\end{array}$ & $\begin{array}{l}0 \% \\
(n=0)\end{array}$ & $\begin{array}{l}0 \% \\
(n=0)\end{array}$ & $\begin{array}{l}0 \% \\
(n=0)\end{array}$ & \multirow[t]{5}{*}{0.136} & $\begin{array}{l}0 \% \\
(n=0)\end{array}$ & $\begin{array}{l}0 \% \\
(n=0)\end{array}$ & $\begin{array}{l}0 \% \\
(n=0)\end{array}$ & \multirow[t]{5}{*}{0.190} & $\begin{array}{l}0.29 \% \\
(n=1)\end{array}$ & $\begin{array}{l}0.88 \% \\
(n=3)\end{array}$ & $\begin{array}{l}0.59 \% \\
(n=2)\end{array}$ & \multirow[t]{5}{*}{0.052} \\
\hline & Disagree & $\begin{array}{l}0 \% \\
(n=0)\end{array}$ & $\begin{array}{l}0 \% \\
(n=0)\end{array}$ & $\begin{array}{l}0 \% \\
(n=0)\end{array}$ & & $\begin{array}{l}0 \% \\
(n=0)\end{array}$ & $\begin{array}{l}5.88 \% \\
(n=1)\end{array}$ & $\begin{array}{l}0 \% \\
(n=0)\end{array}$ & & $\begin{array}{l}1.76 \% \\
(n=6)\end{array}$ & $\begin{array}{l}0.29 \% \\
(n=1)\end{array}$ & $\begin{array}{l}0 \% \\
(n=0)\end{array}$ & \\
\hline & Neutral & $\begin{array}{l}0 \% \\
(n=0)\end{array}$ & $\begin{array}{l}1.57 \% \\
(n=2)\end{array}$ & $\begin{array}{l}0 \% \\
(n=0)\end{array}$ & & $\begin{array}{l}0 \% \\
(n=0)\end{array}$ & $\begin{array}{l}0 \% \\
(n=0)\end{array}$ & $\begin{array}{l}5.88 \% \\
(n=1)\end{array}$ & & $\begin{array}{l}3.81 \% \\
(n=13)\end{array}$ & $\begin{array}{l}1.17 \% \\
(n=4)\end{array}$ & $\begin{array}{l}0.59 \% \\
(n=2)\end{array}$ & \\
\hline & Agree & $\begin{array}{l}00 \% \\
(n=0)\end{array}$ & $\begin{array}{l}27.56 \% \\
(n=35)\end{array}$ & $\begin{array}{l}0 \% \\
(n=0)\end{array}$ & & $\begin{array}{l}23.53 \% \\
(n=4)\end{array}$ & $\begin{array}{l}5.88 \% \\
(n=1)\end{array}$ & $\begin{array}{l}5.88 \% \\
(n=1)\end{array}$ & & $\begin{array}{l}24.05 \% \\
(n=82)\end{array}$ & $\begin{array}{l}10.85 \% \\
(n=37)\end{array}$ & $\begin{array}{l}6.16 \% \\
(n=21)\end{array}$ & \\
\hline & $\begin{array}{l}\text { Strongly } \\
\text { agree }\end{array}$ & $\begin{array}{l}5.51 \% \\
(n=7)\end{array}$ & $\begin{array}{l}59.06 \% \\
(n=75)\end{array}$ & $\begin{array}{l}6.30 \% \\
(n=8)\end{array}$ & & $\begin{array}{l}17.65 \% \\
(n=3)\end{array}$ & $\begin{array}{l}5.88 \% \\
(n=1)\end{array}$ & $\begin{array}{l}29.41 \% \\
(n=5)\end{array}$ & & $\begin{array}{l}21.99 \% \\
(n=75)\end{array}$ & $\begin{array}{l}19.06 \% \\
(n=65)\end{array}$ & $\begin{array}{l}8.50 \% \\
(n=29)\end{array}$ & \\
\hline \multirow{5}{*}{$\begin{array}{l}\text { Guidance on } \\
\text { diet and } \\
\text { lifestyle }\end{array}$} & $\begin{array}{l}\text { Strongly } \\
\text { disagree }\end{array}$ & $\begin{array}{l}0 \% \\
(n=0)\end{array}$ & $\begin{array}{l}0 \% \\
(n=0)\end{array}$ & $\begin{array}{l}0 \% \\
(n=0)\end{array}$ & & $\begin{array}{l}0 \% \\
(n=0)\end{array}$ & $\begin{array}{l}0 \% \\
(n=0)\end{array}$ & $\begin{array}{l}0 \% \\
(n=0)\end{array}$ & & $\begin{array}{l}0.59 \% \\
(n=2)\end{array}$ & $\begin{array}{l}0.88 \% \\
(n=3)\end{array}$ & $\begin{array}{l}0.29 \% \\
(n=1)\end{array}$ & \multirow[t]{5}{*}{0.000} \\
\hline & Disagree & $\begin{array}{l}0 \% \\
(n=0)\end{array}$ & $\begin{array}{l}0 \% \\
(n=0)\end{array}$ & $\begin{array}{l}0 \% \\
(n=0)\end{array}$ & & $\begin{array}{l}0 \% \\
(n=0)\end{array}$ & $\begin{array}{l}0 \% \\
(n=0)\end{array}$ & $\begin{array}{l}0 \% \\
(n=0)\end{array}$ & & $\begin{array}{l}4.41 \% \\
(n=15)\end{array}$ & $\begin{array}{l}0.29 \% \\
(n=1)\end{array}$ & $\begin{array}{l}0.29 \% \\
(n=1)\end{array}$ & \\
\hline & Neutral & $\begin{array}{l}0 \% \\
(n=0)\end{array}$ & $\begin{array}{l}0 \% \\
(n=0)\end{array}$ & $\begin{array}{l}0 \% \\
(n=0)\end{array}$ & & $\begin{array}{l}5.88 \% \\
(n=1)\end{array}$ & $\begin{array}{l}0 \% \\
(n=0)\end{array}$ & $\begin{array}{l}11.76 \% \\
(n=2)\end{array}$ & 0.356 & $\begin{array}{l}10.00 \% \\
(n=34)\end{array}$ & $\begin{array}{l}2.05 \% \\
(n=7)\end{array}$ & $\begin{array}{l}1.76 \% \\
(n=6)\end{array}$ & \\
\hline & Agree & $\begin{array}{l}1.57 \% \\
(n=2)\end{array}$ & $\begin{array}{l}31.50 \% \\
(n=40)\end{array}$ & $\begin{array}{l}0.79 \% \\
(n=1)\end{array}$ & & $\begin{array}{l}29.41 \% \\
(n=5)\end{array}$ & $\begin{array}{l}5.88 \% \\
(n=1)\end{array}$ & $\begin{array}{l}11.76 \% \\
(n=2)\end{array}$ & & $\begin{array}{l}24.71 \% \\
(n=84)\end{array}$ & $\begin{array}{l}9.12 \% \\
(n=31)\end{array}$ & $\begin{array}{l}6.77 \% \\
(n=23)\end{array}$ & \\
\hline & $\begin{array}{l}\text { Strongly } \\
\text { agree }\end{array}$ & $\begin{array}{l}3.94 \% \\
(n=5)\end{array}$ & $\begin{array}{l}56.69 \% \\
(n=72)\end{array}$ & $\begin{array}{l}5.51 \% \\
(n=7)\end{array}$ & 0.389 & $\begin{array}{l}5.88 \% \\
(n=1)\end{array}$ & $\begin{array}{l}11.76 \% \\
(n=2)\end{array}$ & $\begin{array}{l}17.65 \% \\
(n=3)\end{array}$ & & $\begin{array}{l}12.35 \% \\
(n=42)\end{array}$ & $\begin{array}{l}20.00 \% \\
(n=68)\end{array}$ & $\begin{array}{l}6.47 \% \\
(n=22)\end{array}$ & \\
\hline \multirow{5}{*}{$\begin{array}{l}\text { Need for } \\
\text { routine } \\
\text { checkup }\end{array}$} & $\begin{array}{l}\text { Strongly } \\
\text { disagree }\end{array}$ & $\begin{array}{l}0 \% \\
(n=0)\end{array}$ & $\begin{array}{l}0.79 \% \\
(n=1)\end{array}$ & $\begin{array}{l}0.79 \% \\
(n=1)\end{array}$ & \multirow[t]{5}{*}{0.426} & $\begin{array}{l}0 \% \\
(n=0)\end{array}$ & $\begin{array}{l}0 \% \\
(n=0)\end{array}$ & $\begin{array}{l}0 \% \\
(n=0)\end{array}$ & \multirow[t]{5}{*}{0.505} & $\begin{array}{l}0.89 \% \\
(n=3)\end{array}$ & $\begin{array}{l}0.89 \% \\
(n=3)\end{array}$ & $\begin{array}{l}1.48 \% \\
(n=5)\end{array}$ & \multirow[t]{5}{*}{0.000} \\
\hline & Disagree & $\begin{array}{l}0 \% \\
(n=0)\end{array}$ & $\begin{array}{l}0.79 \% \\
(n=1)\end{array}$ & $\begin{array}{l}0 \% \\
(n=0)\end{array}$ & & $\begin{array}{l}0 \% \\
(n=0)\end{array}$ & $\begin{array}{l}0 \% \\
(n=0)\end{array}$ & $\begin{array}{l}0 \% \\
(n=0)\end{array}$ & & $\begin{array}{l}2.37 \% \\
(n=8)\end{array}$ & $\begin{array}{l}1.18 \% \\
(n=) 4\end{array}$ & $\begin{array}{l}0 \% \\
(n=0)\end{array}$ & \\
\hline & Neutral & $\begin{array}{l}0 \% \\
(n=0)\end{array}$ & $\begin{array}{l}0.79 \% \\
(n=1)\end{array}$ & $\begin{array}{l}0 \% \\
(n=0)\end{array}$ & & $\begin{array}{l}11.76 \% \\
(n=2)\end{array}$ & $\begin{array}{l}0 \% \\
(n=0)\end{array}$ & $\begin{array}{l}0 \% \\
(n=0)\end{array}$ & & $\begin{array}{l}5.33 \% \\
(n=18)\end{array}$ & $\begin{array}{l}1.78 \% \\
(n=6)\end{array}$ & $\begin{array}{l}3.55 \% \\
(n=12)\end{array}$ & \\
\hline & Agree & $\begin{array}{l}0.79 \% \\
(n=1)\end{array}$ & $\begin{array}{l}29.37 \% \\
(n=37)\end{array}$ & $\begin{array}{l}1.59 \% \\
(n=2)\end{array}$ & & $\begin{array}{l}11.76 \% \\
(n=2)\end{array}$ & $\begin{array}{l}5.88 \% \\
(n=1)\end{array}$ & $\begin{array}{l}17.65 \% \\
(n=3)\end{array}$ & & $\begin{array}{l}28.40 \% \\
(n=96)\end{array}$ & $\begin{array}{l}8.88 \% \\
(n=30)\end{array}$ & $\begin{array}{l}5.62 \% \\
(n=19)\end{array}$ & \\
\hline & $\begin{array}{l}\text { Strongly } \\
\text { agree }\end{array}$ & $\begin{array}{l}4.76 \% \\
(n=6)\end{array}$ & $\begin{array}{l}56.35 \% \\
(n=71)\end{array}$ & $\begin{array}{l}3.97 \% \\
(n=5)\end{array}$ & & $\begin{array}{l}17.65 \% \\
(n=3)\end{array}$ & $\begin{array}{l}11.76 \% \\
(n=2)\end{array}$ & $\begin{array}{l}23.53 \% \\
(n=4)\end{array}$ & & $\begin{array}{l}15.09 \% \\
(n=51)\end{array}$ & $\begin{array}{l}19.23 \% \\
(n=65)\end{array}$ & $\begin{array}{l}5.32 \% \\
(n=18)\end{array}$ & \\
\hline \multirow{5}{*}{$\begin{array}{l}\text { Adequate } \\
\text { knowledge on } \\
\text { patient } \\
\text { condition }\end{array}$} & $\begin{array}{l}\text { Strongly } \\
\text { disagree }\end{array}$ & $\begin{array}{l}0 \% \\
(n=0)\end{array}$ & $\begin{array}{l}4.65 \% \\
(n=6)\end{array}$ & $\begin{array}{l}0 \% \\
(n=0)\end{array}$ & \multirow[t]{5}{*}{0.946} & $\begin{array}{l}0 \% \\
(n=0)\end{array}$ & $\begin{array}{l}0 \% \\
(n=0)\end{array}$ & $\begin{array}{l}0 \% \\
(n=0)\end{array}$ & \multirow[t]{5}{*}{0.120} & $\begin{array}{l}0.59 \% \\
(n=2)\end{array}$ & $\begin{array}{l}1.76 \% \\
(n=6)\end{array}$ & $\begin{array}{l}1.17 \% \\
(n=4)\end{array}$ & \multirow[t]{5}{*}{0.003} \\
\hline & Disagree & $\begin{array}{l}0 \% \\
(n=0)\end{array}$ & $\begin{array}{l}3.88 \% \\
(n=5)\end{array}$ & $\begin{array}{l}0 \% \\
(n=0)\end{array}$ & & $\begin{array}{l}0 \% \\
(n=0)\end{array}$ & $\begin{array}{l}0 \% \\
(n=0)\end{array}$ & $\begin{array}{l}11.76 \% \\
(n=2)\end{array}$ & & $\begin{array}{l}2.05 \% \\
(n=7)\end{array}$ & $\begin{array}{l}2.05 \% \\
(n=7)\end{array}$ & $\begin{array}{l}1.47 \% \\
(n=5)\end{array}$ & \\
\hline & Neutral & $\begin{array}{l}0 \% \\
(n=0)\end{array}$ & $\begin{array}{l}4.65 \% \\
(n=6)\end{array}$ & $\begin{array}{l}0.78 \% \\
(n=1)\end{array}$ & & $\begin{array}{l}0 \% \\
(n=0)\end{array}$ & $\begin{array}{l}0 \% \\
(n=0)\end{array}$ & $\begin{array}{l}11.76 \% \\
(n=2)\end{array}$ & & $\begin{array}{l}5.57 \% \\
(n=19)\end{array}$ & $\begin{array}{l}2.64 \% \\
(n=9)\end{array}$ & $\begin{array}{l}1.76 \% \\
(n=6)\end{array}$ & \\
\hline & Agree & $\begin{array}{l}3.10 \% \\
(n=4)\end{array}$ & $\begin{array}{l}40.31 \% \\
(n=52)\end{array}$ & $\begin{array}{l}3.10 \% \\
(n=4)\end{array}$ & & $\begin{array}{l}41.18 \% \\
(n=7)\end{array}$ & $\begin{array}{l}17.65 \% \\
(n=3)\end{array}$ & $\begin{array}{l}11.76 \% \\
(n=2)\end{array}$ & & $\begin{array}{l}33.43 \% \\
(n=114)\end{array}$ & $\begin{array}{l}14.08 \% \\
(n=48)\end{array}$ & $\begin{array}{l}6.74 \% \\
(n=23)\end{array}$ & \\
\hline & $\begin{array}{l}\text { Strongly } \\
\text { agree }\end{array}$ & $\begin{array}{l}2.33 \% \\
(n=3)\end{array}$ & $\begin{array}{l}34.88 \% \\
(n=45)\end{array}$ & $\begin{array}{l}2.33 \% \\
(n=3)\end{array}$ & & $\begin{array}{l}0 \% \\
(n=0)\end{array}$ & $\begin{array}{l}0 \% \\
(n=0)\end{array}$ & $\begin{array}{l}5.88 \% \\
(n=1)\end{array}$ & & $\begin{array}{l}10.26 \% \\
(n=35)\end{array}$ & $\begin{array}{l}11.73 \% \\
(n=40)\end{array}$ & $\begin{array}{l}4.69 \% \\
(n=16)\end{array}$ & \\
\hline \multirow{5}{*}{$\begin{array}{l}\text { Fitness and } \\
\text { health } \\
\text { screening }\end{array}$} & $\begin{array}{l}\text { Strongly } \\
\text { disagree }\end{array}$ & $\begin{array}{l}0 \% \\
(n=0)\end{array}$ & $\begin{array}{l}0 \% \\
(n=0)\end{array}$ & $\begin{array}{l}0 \% \\
(n=0)\end{array}$ & \multirow[t]{5}{*}{0.727} & $\begin{array}{l}0 \% \\
(n=0)\end{array}$ & $\begin{array}{l}0 \% \\
(n=0)\end{array}$ & $\begin{array}{l}0 \% \\
(n=0)\end{array}$ & \multirow[t]{5}{*}{0.213} & $\begin{array}{l}1.46 \% \\
(n=5)\end{array}$ & $\begin{array}{l}0 \% \\
(n=0)\end{array}$ & $\begin{array}{l}0.29 \% \\
(n=1)\end{array}$ & \multirow[t]{5}{*}{0.000} \\
\hline & Disagree & $\begin{array}{l}0 \% \\
(n=0)\end{array}$ & $\begin{array}{l}0 \% \\
(n=0)\end{array}$ & $\begin{array}{l}0 \% \\
(n=0)\end{array}$ & & $\begin{array}{l}11.76 \% \\
(n=2)\end{array}$ & $\begin{array}{l}5.88 \% \\
(n=1)\end{array}$ & $\begin{array}{l}0 \% \\
(n=0)\end{array}$ & & $\begin{array}{l}7.60 \% \\
(n=26)\end{array}$ & $\begin{array}{l}0.88 \% \\
(n=3)\end{array}$ & $\begin{array}{l}0.88 \% \\
(n=3)\end{array}$ & \\
\hline & Neutral & $\begin{array}{l}0 \% \\
(n=0)\end{array}$ & $\begin{array}{l}7.75 \% \\
(n=10)\end{array}$ & $\begin{array}{l}0 \% \\
(n=0)\end{array}$ & & $\begin{array}{l}0 \% \\
(n=0)\end{array}$ & $\begin{array}{l}5.88 \% \\
(n=1)\end{array}$ & $\begin{array}{l}0 \% \\
(n=0)\end{array}$ & & $\begin{array}{l}14.62 \% \\
(n=50)\end{array}$ & $\begin{array}{l}2.34 \% \\
(n=8)\end{array}$ & $\begin{array}{l}6.14 \% \\
(n=21)\end{array}$ & \\
\hline & Agree & $\begin{array}{l}3.88 \% \\
(n=5)\end{array}$ & $\begin{array}{l}51.16 \% \\
(n=66)\end{array}$ & $\begin{array}{l}4.65 \% \\
(n=6)\end{array}$ & & $\begin{array}{l}23.53 \% \\
(n=4)\end{array}$ & $\begin{array}{l}5.88 \% \\
(n=1)\end{array}$ & $\begin{array}{l}29.41 \% \\
(n=5)\end{array}$ & & $\begin{array}{l}23.68 \% \\
(n=81)\end{array}$ & $\begin{array}{l}18.13 \% \\
(n=62)\end{array}$ & $\begin{array}{l}6.73 \% \\
(n=23)\end{array}$ & \\
\hline & $\begin{array}{l}\text { Strongly } \\
\text { agree }\end{array}$ & $\begin{array}{l}1.55 \% \\
(n=2)\end{array}$ & $\begin{array}{l}29.46 \% \\
(n=38)\end{array}$ & $\begin{array}{l}1.55 \% \\
(n=2)\end{array}$ & & $\begin{array}{l}5.88 \% \\
(n=1)\end{array}$ & $\begin{array}{l}0 \% \\
(n=0)\end{array}$ & $\begin{array}{l}11.76 \% \\
(n=2)\end{array}$ & & $\begin{array}{l}4.39 \% \\
(n=15)\end{array}$ & $\begin{array}{l}11.11 \% \\
(n=38)\end{array}$ & $\begin{array}{l}1.75 \% \\
(n=6)\end{array}$ & \\
\hline \multirow{2}{*}{$\begin{array}{l}\text { Assist with } \\
\text { welfare } \\
\text { services }\end{array}$} & $\begin{array}{l}\text { Strongly } \\
\text { disagree }\end{array}$ & $\begin{array}{l}0 \% \\
(n=0)\end{array}$ & $\begin{array}{l}3.88 \% \\
(n=5)\end{array}$ & $\begin{array}{l}0 \% \\
(n=0)\end{array}$ & \multirow[t]{2}{*}{0.827} & $\begin{array}{l}0 \% \\
(n=0)\end{array}$ & $\begin{array}{l}0 \% \\
(n=0)\end{array}$ & $\begin{array}{l}0 \% \\
(n=0)\end{array}$ & \multirow[t]{2}{*}{0.687} & $\begin{array}{l}4.18 \% \\
(n=14)\end{array}$ & $\begin{array}{l}1.47 \% \\
(n=5)\end{array}$ & $\begin{array}{l}1.47 \% \\
(n=5)\end{array}$ & \multirow[t]{2}{*}{0.331} \\
\hline & Disagree & $\begin{array}{l}0.78 \% \\
(n=1)\end{array}$ & $\begin{array}{l}10.85 \% \\
(n=14)\end{array}$ & $\begin{array}{l}0 \% \\
(n=0)\end{array}$ & & $\begin{array}{l}5.88 \% \\
(n=1)\end{array}$ & $\begin{array}{l}5.88 \% \\
(n=1)\end{array}$ & $\begin{array}{l}0 \% \\
(n=0)\end{array}$ & & $\begin{array}{l}7.94 \% \\
(n=27)\end{array}$ & $\begin{array}{l}5.88 \% \\
(n=20)\end{array}$ & $\begin{array}{l}2.06 \% \\
(n=7)\end{array}$ & \\
\hline
\end{tabular}




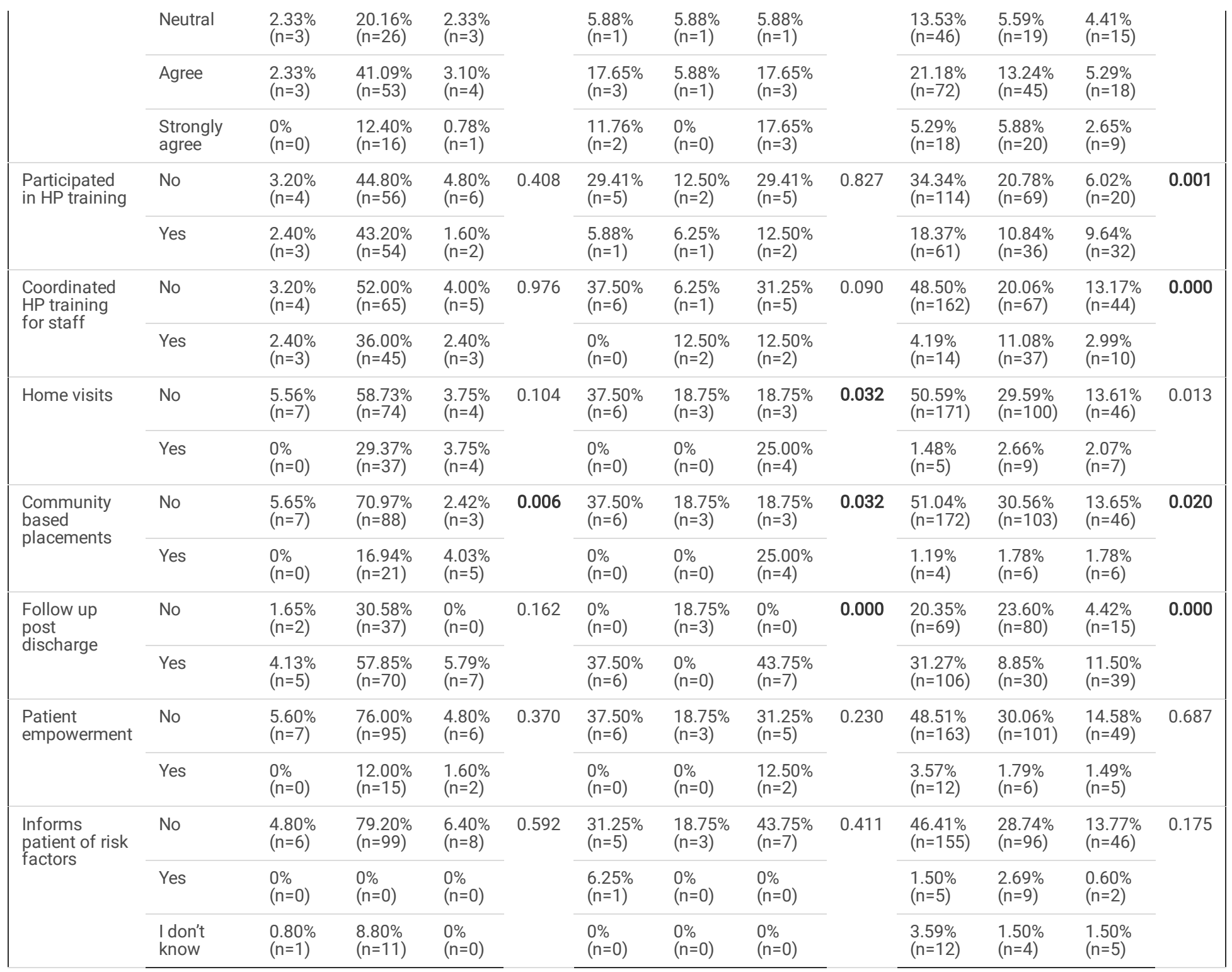

A total of ten HRI dimensions were significantly associated with HP among HCW (Table 4). Among the medical doctors, significant positive predictors of HP among the HRI dimensions were education on disease condition (Coeff: $2.273,95 \% \mathrm{Cl} 1.393$ - 3.710), guidance on diet and lifestyle (Coeff: $0.361,95 \% \mathrm{Cl}$ 0.228 - 0.570), fitness and health screening (Coeff: $0.325,95 \% \mathrm{Cl} 0.224-0.474)$, coordinated HP training for staff (Coeff: $0.216,95 \% \mathrm{Cl} 0.106-0.441)$, home visits (Coeff: $0.141,95 \% \mathrm{Cl} 0.039$ - 0.501), and follow up post discharge (Coeff: $2.743,95 \% \mathrm{Cl} 1.618$ - 4.648) and patient empowerment (Coeff: $3.919,95 \% \mathrm{Cl}$ 1.102 - 13.931). Furthermore, seven significant dimensions predictors of HRI emerged among AHW. These included - education on disease condition (Coeff: 2.117, 95\% Cl 1.107 - 4.048) and community-based placements (Coeff: 3.914, 95\% Cl 1.147 - 13.351) (Table 4). Other dimensions that were significant predictors of HP compared to nurses were need for routine check-up (Coeff: $0.564,95 \% \mathrm{Cl} 0.389-0.818$ ) Fitness and health screening (Coeff: $0.448,95 \% \mathrm{Cl}$ 0.389 - 0.818) and (Coeff: 1.977, 95\% Cl 1.018 - 3.838). The adjusted multivariable model adequately fit the data (Generalized Hosmer-Lemeshow goodnessof-fit test statistic $=17.32, \mathrm{df} .=16, p=0.365)$.

Table 4: Healthcare Workers Related Indicators' dimensions associated with Health Promotion in a multivariate analysis 


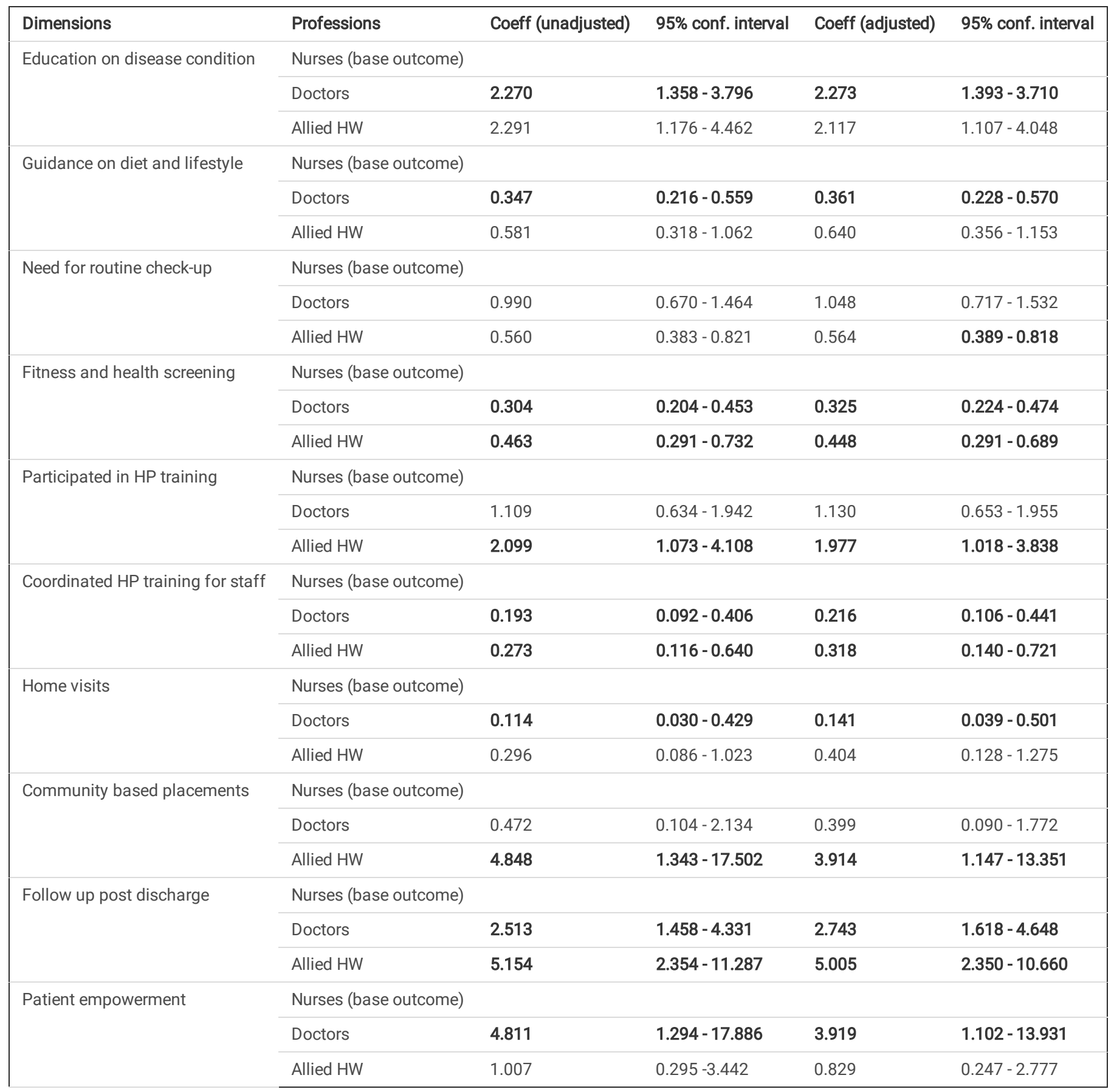

\section{Outcome Related Indicator (ORI)}

Eight indicator dimensions focussing on outcome related indicator were identified. The dimensions that were statistically significant in the study area were reduction of number of diseases, reduction of number of disabilities, and reduced health inequities. Others are improved needs assessments and improved community participation and mobilization (See Additional file 3). Further analysis at the facility levels showed that three indicator dimensions; reduction of number of diseases, reduction of number of disabilities and improved need assessment were statistically significant at the tertiary healthcare level. However, no indicator dimensions were statistically significant at both primary and secondary healthcare levels (Table 5).

Table 5: Correlations of Outcome Related Indicator Dimensions with level of health care Facility 


\begin{tabular}{|c|c|c|c|c|c|c|c|c|c|c|c|c|c|}
\hline \multirow[b]{2}{*}{$\begin{array}{l}\text { ORI } \\
\text { Dimensions }\end{array}$} & \multirow[b]{2}{*}{ Responses } & \multicolumn{4}{|c|}{ Primary Health Care level } & \multicolumn{4}{|c|}{ Secondary Health Care level } & \multicolumn{4}{|c|}{ Tertiary Health care level } \\
\hline & & Doctors & Nurses & AHWs & $\begin{array}{l}p- \\
\text { value }\end{array}$ & Doctors & Nurses & AHWs & $\begin{array}{l}P- \\
\text { value }\end{array}$ & Doctors & Nurses & AHWs & $\begin{array}{l}p- \\
\text { value }\end{array}$ \\
\hline \multirow{2}{*}{$\begin{array}{l}\text { Coverage of } \\
\text { target } \\
\text { population }\end{array}$} & No & $\begin{array}{l}3.96 \% \\
(n=4)\end{array}$ & $\begin{array}{l}33.66 \% \\
(n=34)\end{array}$ & $\begin{array}{l}3.96 \% \\
(n=4)\end{array}$ & 0.171 & $\begin{array}{l}11.11 \% \\
(n=1)\end{array}$ & $\begin{array}{l}22.22 \% \\
(n=2)\end{array}$ & $\begin{array}{l}22.22 \% \\
(n=2)\end{array}$ & 0.325 & $\begin{array}{l}34.05 \% \\
(n=63)\end{array}$ & $\begin{array}{l}23.78 \% \\
(n=44)\end{array}$ & $\begin{array}{l}10.81 \% \\
(n=20)\end{array}$ & 0.440 \\
\hline & Yes & $\begin{array}{l}1.98 \% \\
(n=2)\end{array}$ & $\begin{array}{l}54.55 \% \\
(n=55)\end{array}$ & $\begin{array}{l}1.98 \% \\
(n=2)\end{array}$ & & $\begin{array}{l}22.22 \% \\
(n=2)\end{array}$ & $\begin{array}{l}0.00 \% \\
(n=0)\end{array}$ & $\begin{array}{l}22.22 \% \\
(n=2)\end{array}$ & & $\begin{array}{l}16.76 \% \\
(n=31)\end{array}$ & $\begin{array}{l}8.11 \% \\
(n=15)\end{array}$ & $\begin{array}{l}6.49 \% \\
(n=12)\end{array}$ & \\
\hline \multirow{2}{*}{$\begin{array}{l}\text { Reduction of } \\
\text { number of } \\
\text { deaths }\end{array}$} & No & $\begin{array}{l}4.95 \% \\
(n=5)\end{array}$ & $\begin{array}{l}48.51 \% \\
(n=49)\end{array}$ & $\begin{array}{l}29.70 \% \\
(n=3)\end{array}$ & 0.380 & $\begin{array}{l}0.00 \% \\
(n=0)\end{array}$ & $\begin{array}{l}11.11 \% \\
(n=1)\end{array}$ & $\begin{array}{l}22.22 \% \\
(n=2)\end{array}$ & 0.325 & $\begin{array}{l}20.00 \% \\
(n=38)\end{array}$ & $\begin{array}{l}13.16 \% \\
(n=25)\end{array}$ & $\begin{array}{l}10.53 \% \\
(n=20)\end{array}$ & 0.096 \\
\hline & Yes & $\begin{array}{l}0.99 \% \\
(n=1)\end{array}$ & $\begin{array}{l}39.60 \% \\
(n=40)\end{array}$ & $\begin{array}{l}29.70 \% \\
(n=3)\end{array}$ & & $\begin{array}{l}33.33 \% \\
(n=3)\end{array}$ & $\begin{array}{l}11.11 \% \\
(n=1)\end{array}$ & $\begin{array}{l}22.22 \% \\
(n=2)\end{array}$ & & $\begin{array}{l}30.53 \% \\
(n=58)\end{array}$ & $\begin{array}{l}18.95 \% \\
(n=36)\end{array}$ & $\begin{array}{l}6.84 \% \\
(n=13)\end{array}$ & \\
\hline \multirow{2}{*}{$\begin{array}{l}\text { Reduction of } \\
\text { number of } \\
\text { diseases }\end{array}$} & No & $\begin{array}{l}29.70 \% \\
(n=3)\end{array}$ & $\begin{array}{l}41.58 \% \\
(n=42)\end{array}$ & $\begin{array}{l}29.70 \% \\
(n=3)\end{array}$ & 0.983 & $\begin{array}{l}0.00 \% \\
(n=0)\end{array}$ & $\begin{array}{l}11.11 \% \\
(n=1)\end{array}$ & $\begin{array}{l}33.33 \% \\
(n=3)\end{array}$ & 0.140 & $\begin{array}{l}15.34 \% \\
(n=29)\end{array}$ & $\begin{array}{l}16.40 \% \\
(n=31)\end{array}$ & $\begin{array}{l}10.05 \% \\
(n=19)\end{array}$ & 0.006 \\
\hline & Yes & $\begin{array}{l}29.70 \% \\
(n=3)\end{array}$ & $\begin{array}{l}46.53 \% \\
(n=47)\end{array}$ & $\begin{array}{l}29.70 \% \\
(n=3)\end{array}$ & & $\begin{array}{l}33.33 \% \\
(n=3)\end{array}$ & $\begin{array}{l}11.11 \% \\
(n=1)\end{array}$ & $\begin{array}{l}11.11 \% \\
(n=1)\end{array}$ & & $\begin{array}{l}34.92 \% \\
(n=66)\end{array}$ & $\begin{array}{l}15.87 \% \\
(n=30)\end{array}$ & $\begin{array}{l}7.41 \% \\
(n=14)\end{array}$ & \\
\hline \multirow{2}{*}{$\begin{array}{l}\text { Reduction of } \\
\text { number of } \\
\text { injuries }\end{array}$} & No & $\begin{array}{l}4.95 \% \\
(n=5)\end{array}$ & $\begin{array}{l}59.41 \% \\
(n=60)\end{array}$ & $\begin{array}{l}3.96 \% \\
(n=4)\end{array}$ & 0.717 & $\begin{array}{l}11.11 \% \\
(n=1)\end{array}$ & $\begin{array}{l}11.11 \% \\
(n=1)\end{array}$ & $\begin{array}{l}33.33 \% \\
(n=3)\end{array}$ & 0.539 & $\begin{array}{l}31.74 \% \\
(n=60)\end{array}$ & $\begin{array}{l}16.93 \% \\
(n=32)\end{array}$ & $\begin{array}{l}12.70 \% \\
(n=24)\end{array}$ & 0.138 \\
\hline & Yes & $\begin{array}{l}0.99 \% \\
(n=1)\end{array}$ & $\begin{array}{l}28.71 \% \\
(n=29)\end{array}$ & $\begin{array}{l}1.98 \% \\
(n=2)\end{array}$ & & $\begin{array}{l}22.22 \% \\
(n=2)\end{array}$ & $\begin{array}{l}11.11 \% \\
(n=1)\end{array}$ & $\begin{array}{l}11.11 \% \\
(\mathrm{n}=1)\end{array}$ & & $\begin{array}{l}18.52 \% \\
(n=35)\end{array}$ & $\begin{array}{l}15.34 \% \\
(n=29)\end{array}$ & $\begin{array}{l}4.76 \% \\
(n=9)\end{array}$ & \\
\hline \multirow{2}{*}{$\begin{array}{l}\text { Reduction of } \\
\text { number of } \\
\text { disabilities }\end{array}$} & No & $\begin{array}{l}3.96 \% \\
(n=4)\end{array}$ & $\begin{array}{l}62.38 \% \\
(n=63)\end{array}$ & $\begin{array}{l}1.98 \% \\
(n=2)\end{array}$ & 0.161 & $\begin{array}{l}\begin{array}{l}11.11 \% \\
(n=1)\end{array} \\
\text { a }\end{array}$ & $\begin{array}{l}22.22 \% \\
(n=2)\end{array}$ & $\begin{array}{l}44.44 \% \\
(n=4)\end{array}$ & 0.076 & $\begin{array}{l}28.57 \% \\
(n=54)\end{array}$ & $\begin{array}{l}25.40 \% \\
(n=48)\end{array}$ & $\begin{array}{l}12.70 \% \\
(n=24)\end{array}$ & 0.013 \\
\hline & Yes & $\begin{array}{l}1.98 \% \\
(n=2)\end{array}$ & $\begin{array}{l}25.74 \% \\
(n=26)\end{array}$ & $\begin{array}{l}3.96 \% \\
(n=4)\end{array}$ & & $\begin{array}{l}22.22 \% \\
(n=2)\end{array}$ & $\begin{array}{l}0.00 \% \\
(n=0)\end{array}$ & $\begin{array}{l}0.00 \% \\
(n=0)\end{array}$ & & $\begin{array}{l}21.69 \% \\
(n=41)\end{array}$ & $\begin{array}{l}6.88 \% \\
(n=13)\end{array}$ & $\begin{array}{l}4.76 \% \\
(n=9)\end{array}$ & \\
\hline \multirow[t]{2}{*}{$\begin{array}{l}\text { Reduce health } \\
\text { inequities }\end{array}$} & No & $\begin{array}{l}29.70 \% \\
(n=3)\end{array}$ & $\begin{array}{l}50.50 \% \\
(n=51)\end{array}$ & $\begin{array}{l}4.95 \% \\
(n=5)\end{array}$ & 0.416 & $\begin{array}{l}11.11 \% \\
(n=1)\end{array}$ & $\begin{array}{l}22.22 \% \\
(n=2)\end{array}$ & $\begin{array}{l}44.44 \% \\
(n=4)\end{array}$ & 0.076 & $\begin{array}{l}37.04 \% \\
(n=70)\end{array}$ & $\begin{array}{l}20.63 \% \\
(n=39)\end{array}$ & $\begin{array}{l}14.81 \% \\
(n=28)\end{array}$ & 0.089 \\
\hline & Yes & $\begin{array}{l}29.70 \% \\
(n=3)\end{array}$ & $\begin{array}{l}37.62 \% \\
(n=38)\end{array}$ & $\begin{array}{l}0.99 \% \\
(n=1)\end{array}$ & & $\begin{array}{l}22.22 \% \\
(n=2)\end{array}$ & $\begin{array}{l}0.00 \% \\
(n=0)\end{array}$ & $\begin{array}{l}0.00 \% \\
(n=0)\end{array}$ & & $\begin{array}{l}13.23 \% \\
(n=25)\end{array}$ & $\begin{array}{l}11.64 \% \\
(n=22)\end{array}$ & $\begin{array}{l}2.65 \% \\
(n=5)\end{array}$ & \\
\hline \multirow{2}{*}{$\begin{array}{l}\text { Improved } \\
\text { needs } \\
\text { assessments }\end{array}$} & No & $\begin{array}{l}4.00 \% \\
(n=4)\end{array}$ & $\begin{array}{l}51 \% \\
(n=51)\end{array}$ & $\begin{array}{l}5 \% \\
(n=5)\end{array}$ & 0.444 & $\begin{array}{l}22.22 \% \\
(n=2)\end{array}$ & $\begin{array}{l}0.00 \% \\
(n=0)\end{array}$ & $\begin{array}{l}33.33 \% \\
(n=3)\end{array}$ & 0.196 & $\begin{array}{l}41.27 \% \\
(n=78)\end{array}$ & $\begin{array}{l}20.11 \% \\
(n=38)\end{array}$ & $\begin{array}{l}11.11 \% \\
(n=21)\end{array}$ & 0.012 \\
\hline & Yes & $\begin{array}{l}2 \% \\
(n=2)\end{array}$ & $\begin{array}{l}37 \% \\
(n=37)\end{array}$ & $\begin{array}{l}1 \% \\
(n=1)\end{array}$ & & $\begin{array}{l}11.11 \% \\
(n=1)\end{array}$ & $\begin{array}{l}22.22 \% \\
(n=2)\end{array}$ & $\begin{array}{l}11.11 \% \\
(n=1)\end{array}$ & & $\begin{array}{l}8.99 \% \\
(n=17)\end{array}$ & $\begin{array}{l}12.17 \% \\
(n=23)\end{array}$ & $\begin{array}{l}6.35 \% \\
(n=12)\end{array}$ & \\
\hline \multirow{2}{*}{$\begin{array}{l}\text { Improved } \\
\text { community } \\
\text { participation } \\
\text { and } \\
\text { mobilization }\end{array}$} & No & $\begin{array}{l}4.95 \% \\
(n=5)\end{array}$ & $\begin{array}{l}42.57 \% \\
(n=43)\end{array}$ & $\begin{array}{l}1.98 \% \\
(n=2)\end{array}$ & 0.180 & $\begin{array}{l}11.11 \% \\
(n=1)\end{array}$ & $\begin{array}{l}22.22 \% \\
(n=2)\end{array}$ & $\begin{array}{l}44.44 \% \\
(n=4)\end{array}$ & 0.076 & $\begin{array}{l}37.76 \\
(n=71)\end{array}$ & $\begin{array}{l}24.47 \% \\
(n=46)\end{array}$ & $\begin{array}{l}9.57 \% \\
(n=18)\end{array}$ & 0.099 \\
\hline & Yes & $\begin{array}{l}0.99 \% \\
(n=1)\end{array}$ & $\begin{array}{l}45.54 \% \\
(n=46)\end{array}$ & $\begin{array}{l}3.96 \% \\
(n=4)\end{array}$ & & $\begin{array}{l}22.22 \% \\
(n=2)\end{array}$ & 0 & 0 & & $\begin{array}{l}12.76 \% \\
(n=24)\end{array}$ & $\begin{array}{l}7.98 \% \\
(n=15)\end{array}$ & $\begin{array}{l}7.44 \% \\
(n=14)\end{array}$ & \\
\hline
\end{tabular}

Five dimensions were identified in the unadjusted and adjusted models for medical doctors (Table 6). The significant dimensions for medical doctors include reduction of number of diseases (Coeff $3.151,95 \% \mathrm{Cl} 1.643-6.041$ ), reduction of number of injuries (Coeff $0.412,95 \% \mathrm{Cl} 0.191-0.886$ ), Reduction of number of disabilities (Coeff 4.497, 95\% Cl 2.084 - 9.705), reduced health inequities (Coeff 0.424, 95\% Cl 0.215 - 0.835), and Improved needs assessments (Coeff $0.319,95 \% \mathrm{Cl} 0.164-0.620)$. Among AHWs, 2 predictors each were significant for the unadjusted and adjusted models respectively and include - reduction of number of disabilities, (Coeff $3.522,95 \% \mathrm{Cl} 1.333$ - 9.304), and reduced health inequities (Coeff $0.181,95 \% \mathrm{Cl} 0.063$ - 0.524 ). The generalized HosmerLemeshow test Chi-square was 8.47 with $p$-value 0.933 , indicating a good fit of the final model.

Table 6: Outcome Related Indicators' (ORI) dimension associated with Health Promotion in a multivariate analysis 


\begin{tabular}{|c|c|c|c|c|c|}
\hline \multirow[t]{2}{*}{ ORI Dimensions } & & Coeff (unadjusted) & $95 \%$ conf. interval & Coeff (adjusted) & $95 \%$ conf. interval \\
\hline & Professions & & & & \\
\hline \multirow[t]{3}{*}{ Reduction of number of diseases } & Nurses (base outcome) & & & & \\
\hline & Doctors & 2.869 & $1.466-5.615$ & 3.151 & $1.643-6.041$ \\
\hline & Allied HW & 1.135 & $0.469-2.745$ & 1.014 & $0.439-2.344$ \\
\hline \multirow[t]{3}{*}{ Reduction of number of injuries } & Nurses (base outcome) & & & & \\
\hline & Doctors & 0.391 & $0.177-0.866$ & 0.412 & $0.191-0.886$ \\
\hline & Allied HW & 0.520 & $0.185-1.461$ & 0.535 & $0.199-1.434$ \\
\hline \multirow[t]{3}{*}{ Reduction of number of disabilities } & Nurses (base outcome) & & & & \\
\hline & Doctors & 4.829 & $2.198-10.612$ & 4.497 & $2.084-9.705$ \\
\hline & Allied HW & 3.554 & $1.297-9.739$ & 3.522 & $1.333-9.304$ \\
\hline \multirow[t]{3}{*}{ Reduced health inequities } & Nurses (base outcome) & & & & \\
\hline & Doctors & 0.488 & $0.246-0.969$ & 0.424 & $0.215-0.835$ \\
\hline & Allied HW & 0.145 & $0.046-0.464$ & 0.181 & $0.063-0.524$ \\
\hline \multirow[t]{3}{*}{ Improved needs assessments } & Nurses (base outcome) & & & & \\
\hline & Doctors & 0.378 & $0.189-0.756$ & 0.319 & $0.164-0.620$ \\
\hline & Allied HW & 1.019 & $0.451-2.305$ & 0.986 & $0.454-2.140$ \\
\hline
\end{tabular}

\section{Discussion}

This study was aimed at identifying indicators for HP practice among HCWs from different health care levels in the NMBM, South Africa. We identified three indicator categories that may be used for measuring health promotion (HP) performance of HCWs: (1) facility related indicators (FRI), (2) Healthcare workers related indicators ( $\mathrm{HRI}$ ), and (3) Outcome related indicators (ORI). Association between dimensions and HCWs identified significant FRI dimensions including HP budget, HP communication channel, coordination, and policy and guidelines. Some of the emerging HRI dimensions included guidance on diet \& lifestyle, adequate knowledge on patient condition, follow up and home visits; and ORI dimensions such as reductions in diseases and disabilities, health inequities and improved need assessment. Exploration of relationships between dimensions and various levels of health facilities revealed correlations between HCWs, policy and guidelines at the primary healthcare level. The results further showed association between HCWs and community-based placement (CBP)at the primary level; and HCWs with CBP, home visits and follow up at the secondary level.

The indicators explored in the current study are selected measures or dimensions pointing to adequacy or inadequacy (27) of HP quality. Most respondents in this study reported absence or ignorance of both communication channel and HP coordination in their facilities; The absence of these dimensions could affect the optimal functioning of HP within the health care system. Our results are in concordance with previous reports that have linked the adequate and established channels of communication from within the organization to enhanced health promotion. For instance, Fassl (28) showed that organizations and work environment thrive when leaders adopt effective channels of communication. This also suggests that the success of the organizations and work environment are dependent on the availability of effective leaders that are skilled and confident to transform the vision into reality for improved public health. Hao \& Yazdanifard (29) also concluded that leadership and a clear organization are vital for successful operations (29). To enhance the realization of dimension that improve health promotions, there is need for increased coordination and effective channel of communication. According to Mohr et al (30), enhanced care coordination is largely attributed to favourable patient experiences, cost management and cost effectiveness. This further suggests that FRI has the potential to influence other indicator categories.

Budget was another significant FRI dimension identified in our study. In South Africa, the public health system is funded through the general tax revenue to provide universal coverage to all citizens. Our results showed that participants emphasized the importance of financial allocation through adequate HP budgets. However, most of the respondents reported absence or unawareness of any financial budget for HP activities in the study area. Furthermore, the health system which is dependent on general tax revenue has also been affected by severe austerity measures leading to ineffective functioning of public hospitals. This also may affect the implementation of health promotion policies thus having long term implications on the disease prevention and health promotion outcomes. Financial budget is an all-important driver of health at all levels of care (31-33). This unfavourable response on HP budget may imply a possible lack of structural plan for HP at the policy level or lack of understanding of the potentially accruable benefits from HP.

This study further highlighted low involvement in HP induction training by medical doctors and AHW. The observed low or absence of HP induction training reported by the HCWs may translate to poor HP knowledge by HCWs hence, inadequate HP practices. A recent study by Liu et al (34), which explored the experiences of health-care providers during the COVID-19 crisis in China emphasised the impact of training in enhancing the communication skills and preparedness of HCWs in healthcare management. Furthermore, previous studies reported significant improvement in confidence, knowledge and attitude of employers following a workplace health promotion training programme (35). Health care workers' poor participation in HP training or unawareness of its 
existence in their facilities, may suggest inadequate awareness of HP programmes in the study area. This compares well with findings from a previous study which showed poor HP awareness among HCWs in the facilities (36). However, regarding educating patients on their disease condition, all HCWs fared well in this dimension. This may be because most information communicated to patient in this dimension focuses on biomedical evidence which forms part of the core professional training and not necessarily on HP. Most of the medical doctors and AHW reported absence or ignorance of HP guidelines and policy in their facilities. The observed the poor participation in HP practices among HCWS in the study area may be due to lack of or inadequate HP guidelines and policy. A recent study observed that poor knowledge of HP policy among most HCWs may contribute to poor HP practice among HCWs (37). This emphasizes the need for HCWs to have standardized guideline, policy, and programme appraisal to ensure efficient HP practices by HCWs at all levels.

Findings from the study further showed that HCWs responses regarding home visits to promote patients' health was poor. A previous study showed that most physicians indicated that home care should be provided to patients who are bedridden, incapacitated or patients lacking family support (38). According to these authors, the physicians who participated in the study showed dissatisfaction concerning abuse of services suggesting the need for defined guidelines and policy to protect the interest of both patients and HCWs. Our findings corroborate with a study done in Canada which showed a very good rate of patients' follow-up post discharge (39). However, the Canadian study reported a higher rate of follow-up of patients from bigger urban settings compared to those from the rural areas. The variations observed between home visits and follow-up in our study may suggest that most patients make conscious effort to come back to the hospital, and when they do, they must be attended to. The problem with this is that several patients may be unable to come back to the hospital due to cost, poor health, or other reasons and are therefore excluded from HP care and empowerment. This undermines the goal of Universal Health Care (UHC). For the agenda of UHC to be achieved, there must be a shift from the status quo that focuses only on patients visiting the health care facilities to HCWs visiting patients (40).

Furthermore, reduction in disease and disability were identified as HP outcome measures in the current study. This compares well with a previous study in which Maredza et al (41) showed that the best approach to reduce the burden of stroke is through HP. Reduction in health inequity also emerged as a dimension in measuring HP outcomes and this may be traceable to the historical apartheid background of South Africa that demarcated people along racial lines. The socioeconomic impact of this systemic divide is still evident in the South African healthcare system (42) as the quality of care differs along socioeconomic strata.

To our knowledge, this study is the first to identify HP indicators among healthcare workers in the study area and this is the first time a comparison of HP indicators among HCWs have been compared among different healthcare professionals and at the various levels of health care facilities.

Since this study focused on public hospitals, its findings may have limitations to provide information regarding the private sector. Furthermore, the study was carried out in one municipality and hence the results may not be directly extrapolated to all other municipalities in the country. We therefore recommend further studies to explore the healthcare workers HP practices and perceptions using triangulation methods and possibly in other provinces in South Africa to providing further insight.

\section{Conclusion}

Healthcare systems worldwide are increasingly embracing health promotion as a strategy to attaining universal health coverage. Performance measurement of healthcare workers' health promotion practice using a set of indicators is central to achieving desired goals. Our study has not only identified these indicators but highlighted areas that need improvement. The relevance of individual dimensions classified as FRI, HRI and ORI in this study provides a template for the potentials in healthcare workers to be maximally harnessed. Adoption of these indicators will empower people to take control over their health while reducing cost. Since healthcare workers within health facilities have lasting impact on influencing patients and their relatives' behaviour, this study strives to make a case for HP in healthcare facilities.

We however note that the pathway to institutionalizing measuring indicators may be a long one considering the rigours required of government to address manpower needs, deliberations, setting benchmarks, and defining expected goals for HCWs. This study is therefore a contribution towards initiation of that change process.

\section{Abbreviations}

HP: Health Promotion; HCWs: Healthcare workers; NMBM: Nelson Mandela Bay Municipality, HPCSA: Health Professions Council of South Africa; FRI: Facility relate indicators; HRI: Healthcare worker related indicators; ORI: Outcome related indicators; OECD: Organisation for Economic Co-operation and Development; HP: Health Promotion; LMIC: Low and Middle Income Countries; WHO: World Health Organization; SDG: Sustainable Development Goals; AHW: Allied Health workers; UHC: Universal Health coverage; CBP: community-based placement; NDoH: National Department of Health.

\section{Declarations}

\section{Ethics approval and consent to participate}

The study prior to data collected received ethical approvals from the University of KwaZulu Natal Biomedical Research Ethics committee (BREC), reference BREC /00000088/2019, and the Eastern Cape Health Research committee, reference - EC_201910_012. Gatekeeper approvals were obtained from the respective Healthcare facilities. A written informed consent was received from all the participants before proceeding with the study.

\section{Consent for publication}

Not applicable. 


\section{Availability of data and materials}

The datasets used and/or analysed during the current study are available from the corresponding author on reasonable request.

\section{Competing interests}

The authors declare that they have no competing interests.

\section{Funding}

This study was supported by funding provided by the College of Health Sciences, University of KwaZulu Natal, Durban South Africa. The funding body played no role in the design of the study, analysis, interpretation of the data, or writing of the manuscript.

\section{Authors' contributions}

HM conceived the study idea, conducted proposal writing, data collection and prepared the manuscript. MC is the project supervisor and contributed to subsequent revisions of manuscript. CK analysed the data and contributed to revisions of the manuscript. All authors approved the final manuscript.

\section{Acknowledgements}

I would like to acknowledge the Eastern Cape Health Department, The Nelson Mandela Bay District Health, and the Healthcare workers in the NMB municipality

\section{Author Information}

1 School of Nursing and Public Health, Howard Campus, Desmond Clarence Building, University of KwaZulu Natal, Durban 4001, South Africa.

2 Eastern Cape Department of Health, Livingstone Tertiary Hospital, Stanford Road, Port Elizabeth, 6020, Nelson Mandela Bay, South Africa. South Africa 3 University of Namibia, Katima Mulilo Campus, Box 1096, Katima Mulilo, Namibia

\section{References}

1. Li Y, Ehiri J, Hu D, Oren E, Cao J. Framework of behavioral indicators evaluating TB health promotion outcomes: A modified Delphi study of TB policymakers and health workers. Infect Dis Poverty [Internet]. 2015;4(1). Available from: http://dx.doi.org/10.1186/s40249-015-0087-4

2. Lima RT de S, Fernandes TG, Balieiro AA da S, Costa F dos S, Schramm JM de A, Schweickardt JC, et al. A Atenção Básica no Brasil e o programa Mais Médicos: Uma análise de indicadores de produção. Cienc e Saude Coletiva. 2016;21(9):2685-96.

3. Ader M, Berensson K, Carlsson P, Granath M, Urwitz V. Quality indicators for health promotion programmes. Health Promotion International. 2001.

4. Therezinha R, Oliveira Q De, Henrique A, Moraes A De. Evaluation matrix for health promotion programs in socially vulnerable territories. Cien Saude Colet. 2017;22(12):3915-32.

5. Marco Akerman, Hiram Arroyo, Catherine M. Jones, Michel O'Neill AR and NW. Evaluation of health promotion effectiveness: a political debate and/or a technical exercise? Promot Educ. 2017;14(1_suppl):13-5.

6. Ghebreyesus TA. Urgent health challenges for the next decade [Internet]. World Health Organization. 2020 [cited 2020 Apr 28]. Available from: https://www.who.int/news-room/photo-story/photo-story-detail/urgent-health-challenges-for-the-next-decade

7. Friebe M. Healthcare in need of innovation: exponential technology and biomedical entrepreneurship as solution providers. In: SPIE Digital [Internet]. 2020 [cited 2020 Apr 28]. Available from: https://www.spiedigitallibrary.org/conference-proceedings-of-spie/11315/113150T/Healthcare-in-need-of-innovationexponential-technology-and-biomedical/10.1117/12.2556776.short

8. Woskie L. Crossing the Global Quality Chasm: Improving Health Care Worldwide. National Academies Press; 2018.

9. World Health Organization. THE STATE OF HEALTH in the WHO African Region [Internet]. WHO Regional Office for Africa; 2018. Available from: https://www.afro.who.int/sites/default/files/sessions/documents/State of health in the African Region.pdf

10. Makwero MT. Delivery of primary health care in Malawi. African J Prim Heal Care Fam Med. 2018;10(1):1-3.

11. Olonade O, Olawande TI, Alabi OJ, Imhonopi D. Maternal Mortality and Maternal Health Care in Nigeria: Implications for Socio-Economic Development. 2019;7(5):849-55.

12. Burger R, Christian C. Access to health care in post-apartheid South Africa: availability , affordability , acceptability. 2020;43-55.

13. Maphumulo WT, Bhengu BR. Challenges of quality improvement in the healthcare of South Africa post-apartheid: A critical review. Curationis. 2019;42(1):1-9.

14. Malakoane B, Heunis JC, Chikobvu P, Kigozi NG, Kruger WH. Public health system challenges in the Free State, South Africa: a situation appraisal to inform health system strengthening. 2020;1-14.

15. SAHR. South African Health Review 2019 [Internet]. Health Systems Trust; 2019. Available from: https://www.hst.org.za/publications/South African Health Reviews/SAHR_2019_14012020_Updated web.pdf

16. Conmy A. South African health care system analysis. Public Heal Rev. 2018;1(1).

17. Maseko L, Harris B. People-centeredness in health system reform . Public perceptions of private and public hospitals in South Africa. 2018;48(1):22-7. 
18. Nyasulu J, Pandya H. The effects of coronavirus disease 2019 pandemic on the South African health system: A call to maintain essential health services. African J Prim Heal Care Fam Med. 2020;12(1):1-5.

19. Statistics South Africa. Metropolitan Municipality | Statistics South Africa [Internet]. Statistics South Africa. 2017 [cited 2019 Apr 16]. p. 8-10. Available from: http://www.statssa.gov.za/?page_id=1021\&id=city-of-tshwane-municipality

20. Nelson Mandela Bay Municipality. Nelson Mandela Bay Municipality. Built Environment Performance plan (Draft BEPP) [Internet]. Nelson Mandela Bay Municiopality; 2018. Available from: http://www.nelsonmandelabay.gov.za/datarepository/documents/nmbm-draft-bepp-201819-202021.pdf

21. South Africa History Online (SAHO). Port Elizabeth, Nelson Mandela Bay. 2020; Available from: https://www.sahistory.org.za/place/port-elizabeth-nelsonmandela-bay

22. Nelson Mandela Bay Municipality. Built EnvironmentPerformance Plan Draft BEPP 2018/19 - 2020/21 [Internet]. Journal of Visual Languages \& Computing. Port Elizabeth; 2018. Available from: http://www.nelsonmandelabay.gov.za/datarepository/documents/nmbm-draft-bepp-201819202021.pdf

23. Statistics South Africa. Mid-year population estimates 2019 [Internet]. Pretoria; 2019. Available from: https://www.statssa.gov.za/publications/P0302/P03022019.pdf

24. Abaraogu UO, Onah U, Abaraogu OD, Fawole HO, Kalu ME, Seenan CA. Knowledge, attitudes, and the practice of health promotion among physiotherapists in Nigeria. Physiother Canada. 2019;71(1):92-100.

25. Groene O. Implementing health promotion in hospitals: Manual: Manual and self-assessment forms [Internet]. 2006. Available from: https://apps.who.int/iris/bitstream/handle/10665/107737/E88584.pdf?sequence=1\&isAllowed=y

26. Fagerland M, Hosmer D. A generalized Hosmer-Lemeshow goodness-of-fit test for multinomial logistic regression models. Stata J. 2012;12(3):447-53.

27. Ader M, Berensson K, Carlsson P, Granath M, Urwitz V. Quality indicators for health promotion programmes. Health Promot Int. 2001;16(2):187-95.

28. Fassl M. Internal communication and leadership: the effects on teams' [Internet]. Modul Vienna University; 2018. Available from: https://www.modul.ac.at/uploads/files/Theses/Bachelor/Undergrad_2018/Thesis_1421505_FASSL_Marcel.pdf

29. Hao MJ, Yazdanifard R. How Effective Leadership can Facilitate Change. 2015;15(9):0-6.

30. Mohr DC, Benzer JK, Vimalananda VG, Singer SJ, Meterko M, Mclntosh N, et al. Organizational Coordination and Patient Experiences of Specialty Care Integration. J Gen Intern Med. 2019;34:30-6.

31. Meng Q, Mills A, Wang L, Han Q. What can we learn from China's health system reform? BMJ [Internet]. 2019;365(2349):1-5. Available from: https://www.bmj.com/content/bmj/365/bmj.I2349.full.pdf

32. Stenberg K, Hanssen O, Bertram M, Brindley C, Meshreky A, Barkley S, et al. Articles Guide posts for investment in primary health care and projected resource needs in 67 low-income and middle-income countries: a modelling study. Lancet Glob Heal [Internet]. 2019;7(11):e1500-10. Available from: http://dx.doi.org/10.1016/S2214-109X(19)30416-4

33. Rajan D, Helene B, Stenberg K. Budgeting for health [Internet]. World Health Organization. 2016. Available from: https://apps.who.int/iris/bitstream/handle/10665/250221/9789241549745-chapter8-eng.pdf;jsessionid=FC56BE8FBB5065B44FBEDB6E93FAA929? sequence $=11$

34. Liu Q, Luo D, Haase JE, Guo Q, Wang XQ, Liu S, et al. The experiences of health-care providers during the COVID-19 crisis in China: a qualitative study. Lancet Glob Heal [Internet]. 2020;8(6):e790-8. Available from: http://dx.doi.org/10.1016/S2214-109X(20)30204-7

35. Lang J, Cluff L, Rineer J, Brown D, Jones-jack N. Building Capacity for Workplace Health Promotion: Findings From the Work@Health® Train-the-Trainer Program. Health Promot Pract. 2017;18(6):902-11.

36. Altamimi S, Alshoshan F, Al Shaman G, Tawfeeq N, Alasmary M, Ahmed AE. Health promotion practices as perceived by primary healthcare professionals at the Ministry of National Guard Health Affairs, Saudi Arabia. Qatar Med J. 2016;2016(1):1-9.

37. Arsenijevic J, Groot W. Health promotion policies for elderlySome comparisons across Germany, Italy, the Netherlands and Poland. Health Policy (New York) [Internet]. 2020;(February). Available from: https://doi.org/10.1016/j.healthpol.2020.01.013

38. Aksoy H, Kahveci R, Döner P, Aksoy I, Ayhan D, Koç M, et al. Physicians ' attitudes toward home healthcare services in Turkey: A qualitative study Physicians ' attitudes toward home healthcare services in. 2015;4788.

39. Canadian Institute for Health Information. Physician Follow-Up After Hospital Discharge: Progress in Meeting Best Practices Types of Care. 2015; Available from: https://secure.cihi.ca/free_products/Physician-Follow-Up-Study-mar2015_EN.pdf

40. Nivestam A, Westergren A, Petersson P, Haak M. Factors associated with good health among older persons who received a preventive home visit: A crosssectional study. BMC Public Health. 2020;20(1):1-7.

41. Maredza M, Bertram MY, Gómez-olivé XF, Tollman SM. Burden of stroke attributable to selected lifestyle risk factors in rural South Africa. BMC Public Health [Internet]. 2016; Available from: http://dx.doi.org/10.1186/s12889-016-2805-7

42. Omotoso KO, Koch SF. Assessing changes in social determinants of health inequalities in South Africa: A decomposition analysis. Int $J$ Equity Health [Internet]. 2018;17(1):1-13. Available from: http://dx.doi.org/10.1186/s12939-018-0885-y

\section{Figures}


Consent forms and questionnaires distributed to

520 HCWs in 23 Hospitals

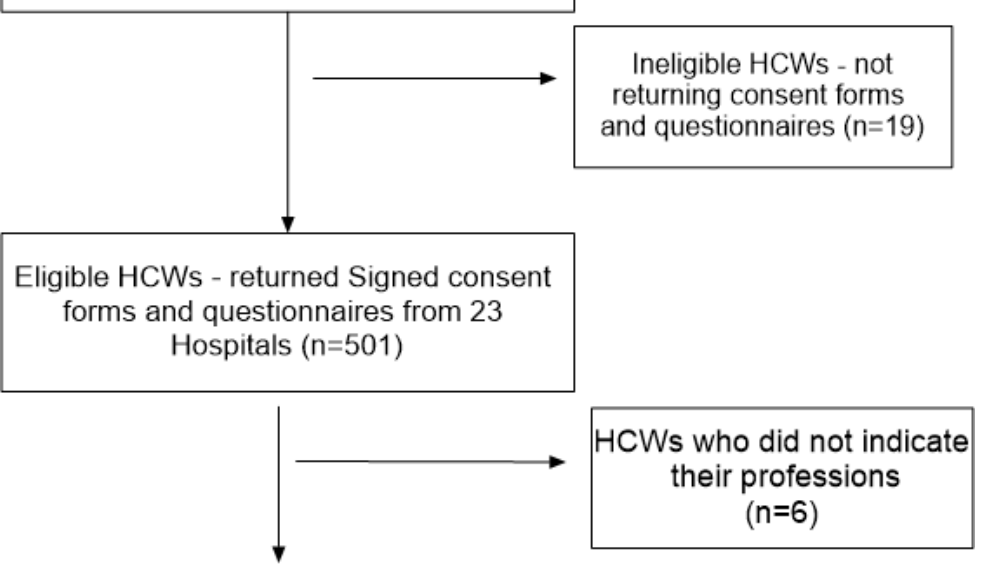

HCWs who indicated their

profession

$(n=495)$

HCWs - Healthcare Workers

Figure 1

Sampling methodology of Healthcare workers in the NMBM

\section{Supplementary Files}

This is a list of supplementary files associated with this preprint. Click to download.

- Additionalfile1.docx

- Additionalfile2.docx

- Additionalfile3.docx 